\title{
Goblet Cell Hyperplasia Increases SARS-CoV-2 Infection in COPD.
}

$11 \quad{ }^{5}$ Lead contact

${ }^{1}$ Department of Biomedical Sciences, University of North Dakota School of Medicine \& Health Sciences, Grand Forks, ND 58202, USA.

${ }^{2}$ Divison of Intramural Research, Rocky Mountain Laboratories, National Institute of Allergy and Infectious Diseases, National Institutes of Health, Hamilton, MT 59840, USA.

${ }^{3}$ Department of Internal Medicine, Pulmonary, Critical Care and Sleep and Allergy, University of Nebraska Medical Center, Omaha, NE 68198, USA.

${ }^{4}$ Contributed equally to this study

*Correspondence: masfique.mehedi@und.edu

Summary. SARS-CoV-2 has become a major problem across the globe, with approximately 50 million cases and more than 1 million deaths and currently no approved treatment or vaccine. Chronic obstructive pulmonary disease (COPD) is one of the underlying conditions in adults of any age that place them at risk for developing severe illness associated with COVID-19. We established an airway epithelium model to study SARS-CoV-2 infection in healthy and COPD lung cells. We found that both the entry receptor ACE2 and the co-factor transmembrane protease TMPRSS2 are expressed at higher levels on nonciliated goblet cell, a novel target for SARS-CoV-2 infection. We observed that SARS-CoV-2 infected goblet cells and induced syncytium formation and cell sloughing. We also found that SARS-CoV-2 replication was 
increased in the COPD airway epithelium likely due to COPD associated goblet cell hyperplasia. Our results reveal goblet cells play a critical role in SARS-CoV-2 infection in the lung.

Keywords. SARS-CoV-2, COVID-19, goblet cells, ciliated cells, COPD, squamous metaplasia, air-liquid interface, syncytium, cell sloughing, goblet cell hyperplasia.

Introduction. Severe acute respiratory syndrome coronavirus 2 (SARS-CoV-2, a causative agent of coronavirus disease 2019, COVID-19) that emerged in December 2019 in Wuhan, China. Since then, this pathogen has caused havoc in the healthcare systems worldwide and consequentially ravaged the economy of countries with COVID-19 outbreaks. There is currently no FDA-approved vaccine against SARS-CoV-2. SARS-CoV-2 is a nonsegmented, positivesense, single-strand RNA virus that causes both upper and lower respiratory tract infections. Most patients exhibit fever and cough, and a subset of patients advance to severe acute respiratory distress syndrome (ARDS) (Guan et al., 2020; Yang et al., 2020). Therefore, patients with underlying chronic obstructive pulmonary disease (COPD) are vulnerable to COVID-19, and in fact, COPD is one of the high-risk factors for severe illness associated with COVID-19 (CDC, 2020; Leung et al., 2020; Sin, 2020).

Viral infections begin by the attachment of viral particles to entry receptors on the host cell. The tissue expression and distribution of the SARS-CoV-2 entry receptor angiotensinconverting enzyme 2 (ACE2) and its co-factor transmembrane serine protease 2 (TMPRSS2) determine the tropism of virus infection (Hoffmann et al., 2020; Li et al., 2003), and viral infection in human airway epithelium depends on ACE2 expression (Hamming et al., 2004; Jia et al., 2006). For successful entry into cells, SARS-CoV-2 uses the serine protease TMPRSS2 for S 
protein priming (Hoffmann et al., 2020). ACE2 is highly expressed in the small intestine, testis, kidneys, heart, thyroid, and adipose tissue and is expressed at moderate expression levels in the lung, colon, liver, bladder, and adrenal gland; and lowest in the blood, spleen, bone marrow, brain, blood vessels, and muscle (Hamming et al., 2004; Li et al., 2020). ACE2 expression in the lungs is predominantly observed in alveolar type 2 (AT2) cells (Lukassen et al., 2020; Qi et al., 2020; To and Lo, 2004; Ziegler et al., 2020), but ciliated cells also express ACE2 in the respiratory epithelium (Sims et al., 2005). Recent RNAseq-based studies have suggested that ACE2 is more highly expressed on goblet cells in the nasal airways and on secretory cells in subsegmental bronchial branches of the lung (Lukassen et al., 2020; Sungnak et al., 2020; Ziegler et al., 2020). Although ACE2 and TMPRSS2 expressions are higher in nonciliated goblet cells compared to ciliated cells (Lukassen et al., 2020; Sungnak et al., 2020; Zhang et al., 2020; Ziegler et al., 2020), it appears that goblet cells are underappreciated in the SARS-CoV-2 infection studies. The possibility that SARS-CoV-2 infects goblet cells could explain the presence of viral RNA in sputum (Wang et al., 2020) and might explain the efficient transmission of the virus from person to person (Dhand and Li, 2020; Wolfel et al., 2020). Importantly, goblet cell hyperplasia is a characteristic pathological feature of COPD patients, who are vulnerable to severe disease associated with COVID-19 (Lippi and Henry, 2020; Shimura et al., 1996; Zhao et al., 2020). Therefore, it is prudent to determine to what extent SARS-CoV-2 infects goblet cells in the lung.

To determine the expression of the SARS-CoV-2 receptor and its preferential cell tropism in the lung, we developed an in vitro airway epithelium model by differentiating primary normal human bronchial (NHBE) cells derived from either a patient with COPD or a healthy adult (nonCOPD). The COPD airway epithelium model recapitulates many bronchial characteristics of 
COPD. We evaluated the expression of ACE2 and TMPRSS2 and studied SARS-CoV-2 infection in these in vitro airway epithelium models. We found that SARS-CoV-2 primarily infects nonciliated goblet cells due to high expression of both ACE2 and TMPRSS2 in these cells. Goblet cell hyperplasia increases of SARS-CoV-2 infection in the COPD airway epithelium. Thus, SARS-CoV-2 targeting and replication in goblet cells may explain the development of more severe COVID-19 in COPD patients.

\section{Results.}

\section{The airway epithelium model recapitulates the chronic bronchial characteristics of COPD.}

We first established an in vitro airway epithelium model by differentiating NHBE cells from either a healthy adult or a COPD patient (deidentified) at the air-liquid interface (ALI). We found that 4 weeks of differentiation provides a fully differentiated pseudostratified mucociliary airway epithelium for both conditions (Figures 1A and B), which allows the use of this model for sideby-side comparisons. NHBE cells from a healthy adult differentiated primarily into a pseudostratified columnar epithelium, whereas the NHBE cells from a COPD patient differentiated into a mix of pseudostratified and stratified columnar epithelium that contained all three main cell types of the respiratory epithelium (Pawlina, 2016; Rayner et al., 2019; Rigden et al., 2016): ciliated cells, goblet cells, and basal cells (Figure 1B). We found that the apical site of the epithelium mainly consists of ciliated and nonciliated goblet cells (Figures 1C and D). Studies have shown that mucin 5AC (MUC5AC) is predominately expressed by airway goblet cells and that mucin 5B (MUC5B) is expressed by goblet cells of submucosal glands (Whitsett, 2018). Club cells are the progenitors of goblet cells, which might express both MUC5AC and MUC5B (Kiyokawa and Morimoto, 2020; Okuda et al., 2019). As expected, we found 

heterogenicity in the cell population with differential expression of common goblet cell markers (Figure 1E) (Lukassen et al., 2020). Basal cells are known for their self-renewal property and give rise to multiple types of differentiated airway epithelial cells (Crystal, 2014). For the detection of basal cells, we sectioned the epithelium and stained the sections for the basal cell marker P63 (Persson et al., 2014; Wang et al., 2002). We found that basal cells reside at the basal membrane of both epithelia and that the COPD epithelium has more basal cells than the healthy epithelium as it is known from respiratory epithelium of COPD patients (Figure 1F) (Higham et al., 2019; Polosukhin et al., 2011).

COPD is associated with abnormal airway and alveolar responses during exposure to noxious stimuli (Brusselle et al., 2011). Because our COPD airway epithelium model was differentiated from NHBE cells, it should recapitulate the bronchial airway phenotypes instead of the alveolar phenotype as more commonly associated with emphysema (Barnes, 2013). To determine whether our in vitro COPD airway epithelium model recapitulates some of the bronchial pathophysiological characteristics of COPD, we focused on two different aspects: goblet cell hyperplasia and squamous metaplasia. First, we compared the number of MUC5 $\mathrm{AC}^{+}$ or MUC5B ${ }^{+}$goblet cells between healthy and COPD epithelia. We found a higher ratio of goblet cells in the COPD airway epithelium (Figures $1 \mathrm{G}$ and $\mathrm{H}$ ). The higher number of goblet cells in the COPD epithelium suggests a persistent goblet cell differentiation, which results in goblet cell hyperplasia (Kim et al., 2015; Reid et al., 2018; Shaykhiev, 2019). Indeed, we found a patch of higher number of goblet cells with extensive mucus secretion in the COPD epithelium (Figure 1I, center). We also found an apparent loss of pseudostratified epithelium accompanied by squamous metaplasia in the COPD epithelium, which is a common pathological phenotype in COPD (Figure 1I, right) (Rigden et al., 2016). 
To determine the biophysical properties of these respiratory epithelia, we assessed the

116

117

118

119

120

121

122

123

124

125

126

127

128

129

130

131

132

133

134

135

136

ACE2 and TMPRSS2 are expressed at higher levels in goblet cells. SARS-CoV-2 infects the tissue membrane integrity (transepithelial electrical resistance, TEER) and ciliary function (ciliary beat frequency, CBF) and found no significant difference in these biophysical properties between healthy and COPD epithelia (Figures $1 \mathrm{~J}$ and $\mathrm{K}$ ). These results indicated that NHBE cells from patients with COPD produced a mix of pseudostratified and stratified highly differentiated mucociliary epithelium with appropriate biophysical properties.

human airway epithelium, and virus entry depends on the host cell receptors ACE2 and its cofactor TMPRSS2 (Hoffmann et al., 2020). We quantified the ACE2 transcript levels in a lung epithelial cell line (A549 cells) and primary NHBE cells in a monolayer or in the differentiated airway epithelium by real-time PCR. We did not detect ACE2 transcripts in the A549 cells (Figure S1A), which might indicate low or no expression of ACE2 confirming previous data (Blanco-Melo et al., 2020; Harcourt et al., 2020; Hoffmann et al., 2020; Jia et al., 2005). However, we detected ACE2 transcripts in primary NHBE cells in both the monolayer and differentiated airway epithelium (Figure S1A). Despite their similar biophysical properties (tissue barrier integrity and ciliary function, Figures $1 \mathrm{~J}$ and $\mathrm{K}$, respectively), ACE2 expression was higher in the COPD epithelium compared to those derived from a healthy donor (Figures S1A-C). We then visualized the expression of ACE2 in the airway epithelium by immunofluorescence imaging. We observed ACE2 expression in both the healthy and COPD airway epithelia and found that ACE2 expression hardly overlapped with cilia on the apical site of the epithelium (Figures 2A and S1D). Nevertheless, low levels of ACE2 expression were 
137 observed on ciliated cells in our model (Figure S1E). These results suggest that the SARS-CoV-2

138 entry receptor ACE2 is mainly expressed on non-ciliated cells in the respiratory epithelium.

Because ACE2 staining hardly overlapped with acetyl-alpha-tubulin, we tested the

140 expression of ACE2 along with that of the goblet and club cell markers MUC5AC and MUC5B

141 (Lukassen et al., 2020). Indeed, ACE2 overlapped with MUC5AC (Figure 2B) and MUC5B

142 (Figures 2C and S1F). We also compared ACE2 expression with the expression of E-cadherin

143 and Zonula occludens-1 (ZO-1), markers for adherens junction and tight junction proteins,

144 respectively. The results showed that ACE2 expression did not overlap with apical tight

145 junctions or adherent junctions (Figure S2), which suggests that ACE2 expression is primarily

146 located within the cellular boundary and does not impact the tissue barrier integrity of the

147 respiratory epithelium. Overall, ACE2 expression was higher in the COPD than in the healthy

148 epithelium (Figures 2 and S1-2), which is likely due to the presence of goblet cell hyperplasia in

149 the COPD epithelium and thus a higher number of goblet cells.

TMPRSS2 is an important host co-factor for SARS-CoV-2 entry into target cells

152 (Hoffmann et al., 2020; Lukassen et al., 2020; Shulla et al., 2011). We visualized TMPRSS2 in

153 the apical site of the airway epithelium by staining with anti-TMPRSS2 and found that

154 TMPRSS2 expression hardly overlapped with cilium (Figure 3A). We then tested the expression

155 of TMPRSS2 along with that of the goblet cell markers MUC5AC and MUC5B. Indeed,

156 TMPRSS2 overlapped with MUC5AC (Figure 3B) and MUC5B (Figures 3C). Therefore, it

157 appears that both TMPRSS2 and ACE2 are expressed on the same cell surface (Figures 2 and 3).

158 These results indicate that goblet cells may be a novel target of SARS-CoV-2 infection in the

159 respiratory epithelium. 
161 SARS-CoV-2 infects goblet cells. Although we confirmed that SARS-CoV-2 entry receptors are

162

163

164

165

166

167

168

169

170

171

172

173

174

175

176

177

178

179

180

181

expressed at higher levels on nonciliated goblet cells, a number of previous studies have suggested that SARS-CoV-2 targets ciliated cells (Hou et al., 2020; Lamers et al., 2020). Therefore, we first examined whether SARS-CoV-2 infects nonciliated goblet cells. We infected the apical side of the airway epithelium with SARS-CoV-2 at a multiplicity of infection of 0.1 $(\mathrm{MOI}=0.1)$. At 4 days post infection (DPI), we fixed the cells and stained them for SARS-CoV2 nucleoprotein $(\mathrm{N})$ and a ciliated cell marker. The results revealed that SARS-CoV-2 infects both healthy and COPD epithelium and causes a substantial cytopathic effect (CPE) (Figures S3A and B). SARS-CoV-2 infection was higher in the COPD epithelium than in the healthy epithelium, as will be addressed later in the manuscript. Although in some cases the virusinduced extensive CPE made it difficult to distinguish SARS-CoV-2 cell tropism, we focused on multiple random areas with less CPE but virus infection. We found that SARS-CoV-2 infects nonciliated cells in both healthy and COPD epithelium (Figure 4A). We also used a second detection method to visualize viral and cellular markers in cross sections of the epithelium. Immunohistochemistry-based staining confirmed the extensive CPE induced by SARS-CoV-2 in both healthy and COPD epithelium. Apparently, SARS-CoV-2 infected both ciliated and nonciliated epithelial cells in the airway epithelium (Figure 4B). Using staining strategies similar to those described before, we found that SARS-CoV-2 infects MUC5B-positive (Figures 4C and D) and MUC5AC-postive (Figure 4E) goblet cells. To determine whether SARS-CoV-2 infects basal cells, we stained the sectioned epithelium for P63 and the SARS-CoV-2 spike (S) protein. We did not observe any overlap between the SARS-CoV-2 S protein and the basal cell marker 
182 (Figure S4). These results suggest that SARS-CoV-2 infects nonciliated goblet cells in addition

183 to ciliated cells.

184

185 SARS-CoV-2 induces syncytia and cell sloughing in the airway epithelium. To determine

186 whether SARS-CoV-2 infection in the airway epithelium recapitulates the virus-induced

187 pathophysiology in the lung, we examined the infected epithelium under a confocal microscope

188 and found that SARS-CoV-2 infection causes substantial damage to the apical site of the infected

189 epithelium, as confirmed by extensive CPE in both healthy and COPD epithelium (Figure S3).

190 We also found substantial mucus secretion due to SARS-CoV-2 infection. The apical damage of

191 the SARS-CoV-2-infected epithelium included loss of cellular junctions, loss of ciliary damage,

192 substantial mucus production, and the protraction of nuclei, which are all common features

193 observed in SARS-CoV-2-infected lungs (Schaefer et al., 2020). Additionally, we investigated

194 whether SARS-CoV-2-infected cells in the epithelium might form syncytium (multinucleated

195 cell), a hallmark of SARS-CoV-2 infection in the lung (Giacca et al., 2020) that had also been

196 reported for SARS-CoV-1 (Franks et al., 2003). Indeed, we found that SARS-CoV-2-infected

197 cells formed syncytia in both healthy and COPD epithelia (Figure 5A and B). Cell sloughing has

198 been reported from lung autopsy findings of SARS-CoV-2-infected patients (Schaefer et al.,

199 2020). Therefore, we examined whether SARS-CoV-2 infection in our epithelium model

200 recapitulates cell sloughing. Indeed, we found that SARS-CoV-2 induces cell sloughing in both

201 healthy and COPD epithelia as confirmed by two independent methods, immunofluorescence

202 and IHC (Figures 5C and D). These results demonstrate that hallmark pathological features of

203 SARS-CoV-2 are recapitulated in the infected airway epithelium model. 
SARS-CoV-2 replicates higher and exacerbates pathophysiology in COPD epithelium. To determine whether SARS-CoV-2 replicates better in the COPD epithelium, we titered SARS$\mathrm{CoV}-2$ in the apical wash of the infected epithelium and found that SARS-CoV-2 replication was increased by almost a log in the COPD compared to the healthy epithelium (Figure 6A). In addition, we found a squamous metaplasia in SARS-CoV-2-infected COPD epithelium which was rather infrequently found in the SARS-CoV-2-infected healthy epithelium (Figures 1I; 4B, D, and E, and 5B and D) (Borczuk et al., 2020). Squamous metaplasia is known to increase

212 bronchial wall thickening as seen in bronchitis (Randell, 2006; Reid et al., 2018; Rigden et al., 213 2016). As tracheobronchitis is one of the most common histopathological features in the COVID-19 disease fatalities (Martines et al., 2020), we evaluated whether SARS-CoV-2

215 infection increases height of the epithelium. First, there was a substantial increase in squamous metaplasia in the COPD epithelium due to SARS-CoV-2 infection (Figure S5). Second, the

217 increased metaplasia apparently changed the morphology of the nonciliated goblet cells in the 218 infected COPD epithelium (Figures 6B and C). Whether this change in the goblet cell morphology impacted the mucus hyperplasia remains to be determined. Third, in contrast to the healthy epithelium, SARS-CoV-2 induced higher squamous metaplasia in the infected COPD epithelium and caused a substantial increase in the height of the epithelium (Figure 6D). These results suggest that SARS-CoV-2 replicates better in the COPD epithelium and exacerbates pathophysiology in the infected airway epithelium.

\section{Discussion.}

Here, we have shown that the COPD epithelium model recapitulates the bronchial 
squamous metaplasia (Gohy et al., 2019; Kim et al., 2015; Reid et al., 2018; Rigden et al., 2016). A previous report suggested the presence of altered ciliated cells in COPD airway epithelium (Gohy et al., 2019), which was not observed in our model. The duration of cell differentiation may be the reason, as we differentiated NHBE cells for four weeks that may require to see terminal differentiation (including ciliogenesis) (Gohy et al., 2019). We used an air-liquid interface (ALI) culture method to generate multi-cellular diversity and physiologic functioning airway epithelium that resembles the airway surface in vivo (Fulcher et al., 2005; Pawlina, 2016; Rayner et al., 2019). One of the limitations in the ALI culture research is passaging of primary NHBE cells may impact on their ability to differentiate into airway epithelium. We could demonstrate that primary NHBE cells obtained after four passages without using any additional supplements can still be differentiated into human airway epithelium (Rayner et al., 2019). In a separate study, we confirmed that passaging NHBE cells up to four times has insignificant effect on the whole-genome transcriptome by comparing transcriptome profiles of each passage cells (data not shown). The ability to expand primary cells that also form fully differentiated mucociliary epithelium reduces repeat sample collections from patients where samples are difficult and limited, such as infants and deceased patients (Rayner et al., 2019; Wolf et al., 2017). Our results demonstrate primary NHBE cells either from healthy or a COPD patient can be passaged up to four times and that normal epithelial phenotypic features are maintained in passaged primary NHBE cells.

One emergent question is why the human-to-human transmission of SARS-CoV-2 is much higher compared to SARS-CoV-1, although both viruses share ACE2 as cell surface receptor and use TMPRSS2 to facilitate their entry into the host cell (Hoffmann et al., 2020; Lukassen et al., 2020). The SARS-CoV-2 spike protein has an additional furin cleavage site that 
is absent in SARS-CoV-1, and it is hypothesized that furin cleavage facilitates human-to-human transmission (Coutard et al., 2020; Lukassen et al., 2020). We found that SARS-CoV-2 infected both ciliated cells and nonciliated goblet cells, but not basal cells in the airway epithelium. Although SARS-CoV-2 may preferentially infect goblet cells due to the higher expression of ACE2 and TMPRSS2, further studies are needed to confirm temporal and spatial regulations of SARS-CoV-2 infection in the airway epithelium. The major function of goblet cells in the lung epithelium is mucin production to trap pathogens, dust, and particles, which are cleared by a process known as mucociliary clearance (Rogers, 2003). The possibility that SARS-CoV-2 infects goblet cells could explain the presence of viral RNA in sputum (Wang et al., 2020) and might explain the easy transmission of the virus from person to person. While we are preparing our manuscript, Hao et al., have shown that goblet cells are permissive to SARS-CoV-2 infection (Hao et al., 2020). As SARS-CoV-1 infection is limited to ciliated cells (Sims et al., 2005), we think that SARS-CoV-2 infection in goblet cells could explain why SARS-CoV-2 is more transmissible than SARS-CoV-1. Influenza A virus infects goblet cells in addition to ciliated cells (Matrosovich et al., 2004), whereas respiratory syncytial virus (RSV) infects only ciliated cells (Zhang et al., 2002). Therefore, virus-specific preferential cell tropism in the lung could explain the difference in respiratory virus pathogenicity and transmissibility.

Our airway epithelium model recapitulated some SARS-CoV-2-induced lung histopathological findings, such as prominent nucleoli (Schaefer et al., 2020), cytoplasmic vacuolation, multinucleated giant cells (Falasca et al., 2020), squamous metaplasia (Martines et al., 2020) and epithelial cell sloughing, which is the most common finding (Bradley et al., 2020). SARS-CoV-2 infection in the bronchial epithelium models induced a substantial amount of cell syncytium formation and cell sloughing, which indicates that tracheobronchial cells are highly 
associated with virus-induced pathogenesis. Therefore, our data suggest that bronchial cells are equally important to alveolar cells in the SARS-CoV-2 pathogenesis (Martines et al., 2020). In patients with COPD, goblet cell hyperplasia is a common clinical manifestation, and an increasing number of goblet cells results in more mucus production (Shaykhiev, 2019). Because we have shown that SARS-CoV-2 replicated at a higher rate in the COPD epithelium, we believe that goblet cell hyperplasia is responsible for this phenomenon and may explain why COPD patients are at higher risk for severe outcome of COVID-19. Interestingly, we found that SARSCoV-2 infection induced "squamous metaplasia" in the COPD epithelium. Squamous metaplasia in the columnar epithelium corelates with increased severity of airway obstruction in COPD (Araya et al., 2007). Although squamous metaplasia is one of the histopathological findings in the SARS-CoV-2 infected patient fatalities (Martines et al., 2020), our results provide evidence for the first time that SARS-CoV-2 infection increases squamous metaplasia, which may result in bronchitis in the infected COPD patients. In fact, tracheobronchitis is one of the most common histopathological features in the COVID-19 disease fatalities (Martines et al., 2020). No SARSCoV-2 infectious virion was detected in the basal medium of the infected healthy and COPD epithelia, which might suggest that the initial virus-induced substantial damage was limited at the apical site.

Immune responses to viral infection play a critical role in determining the clinical outcomes of SARS-CoV-2 infection (Huang et al., 2020). Unfortunately, our airway epithelium model lacks both resident and infiltrating immune cells (dendritic cells, macrophages, and lymphocytes, etc.) and is devoid of the endothelial layer, and therefore cannot study the impact of immune responses on the SARS-CoV-2 infections. However, we can provide inferences based on the detection of immunoregulatory factors (chemokines and cytokines), but these hypotheses 
remain to be confirmed. Overall, we believe that the airway epithelium model provides an excellent tool for demystifying some of the SARS-CoV-2 pathophysiological features and identifying and testing novel therapeutics against the virus.

302 infection of goblet cells leading to virus-induced syncytium formation and cell sloughing in the airway epithelium. We found that SARS-CoV-2 replicates better COPD airway epithelium likely due to COPD associated goblet cell hyperplasia. Thus, we postulate that goblet cells play a critical role in SARS-CoV-2 infection of the lung and are responsible for more severe outcome of SARS-CoV-2 infection in COPD patients.

Figure legends. adult or COPD patient were fixed, embedded in paraffin, sectioned and stained with hematoxylin

312 and eosin $(\mathrm{H} \& \mathrm{E})$. The nuclei are stained dark-purple, and the cytoplasmic components are pink.

313 Bar $=50 \mu \mathrm{m}$. (B). The sectioned epithelia were stained with Alcian blue. Mucosubstances are

314 stained blue, whereas the cytoplasmic components are pale pink, and the nuclei are pink/red. Bar $315=50 \mu \mathrm{m} .(\mathbf{C})$. The apical sites of the airway epithelia were fixed, permeabilized and stained for 316 the goblet cell marker MUC5AC (anti-MUC5AC, green), the ciliated cell marker acetyl-alpha 317 tubulin (anti-acetyl-alpha tubulin, cyan), and F-actin (rhodamine phalloidin, red); the nuclei were 318 also stained with DAPI (blue). Bar $=10 \mu \mathrm{m}$. (D). The sectioned epithelia were stained for the 319 ciliated cell marker acetyl-alpha tubulin (anti-acetyl-alpha tubulin, cyan), the goblet cell marker 
MUC5AC (anti-MUC5AC, green), and F-actin (rhodamine phalloidin, red); the nuclei were also stained with DAPI (blue). Bar $=20 \mu \mathrm{m}$. (E). The apical sites of the airway epithelia were fixed and stained for two goblet cell markers, MUC5AC and MUC5B [anti-MUC5AC (green) and anti-MUC5B (cyan), respectively] and F-actin (rhodamine phalloidin, red); the nuclei were also stained with DAPI (blue). Bar $=10 \mu \mathrm{m}$. (F). The sectioned epithelia were stained for the basal cell marker P63 (anti-P63, green), the goblet cell marker MUC5B (anti-MUC5B, cyan), and Factin (rhodamine phalloidin, red); the nuclei were also stained with DAPI (blue). Bar $=20 \mu \mathrm{m}$. (G). The MUC5B ${ }^{+}$goblet cells were counted from tiled images of the airway epithelium stained for MUC5B (anti-MUC5B, cyan) (described above) obtained with a Leica DMi8 epifluorescence microscope. A total of approximately 3,500 cells were counted from each epithelium to 330 determine the ratio. (H). The $\mathrm{MUC5} \mathrm{AC}^{+}$goblet cells were counted from random fields in 331 confocal images of the airway epithelium stained for MUC5AC (anti-MUCAC, cyan) (described above). A total of approximately 1,500 cells were counted from each epithelium to determine the ratio. (I). The sectioned epithelia were stained for MUC5B (anti-MUC5B, cyan); the nuclei were stained with DAPI (blue). Bar $=20 \mu \mathrm{m}$. (J). Transepithelial electrical measurements (TEERs) of the airway epithelia were obtained. The data were obtained by combining three independent Transwell reads, and each Transwell read was an average of three independent reads. The error bars represent the SEMs. (K). The ciliary beat frequency $(\mathrm{CBF})$ was measured on the airway epithelia. The data were obtained by combining three independent Transwell reads, and each

339 Transwell read was an average of six random point reads. The error bars represent the SEMs.

340 The statistical significance was determined by unpaired two-tailed t-tests. The results from one 341 independent experiment are shown. 
Figure 2. ACE2 is expressed at higher levels in goblet cells. The airway epithelia were generated and fixed as described in Figure 1 (A) Cells were stained for ACE2 (anti-ACE2, green), cilia (anti-acetyl-alpha tubulin, cyan) F-actin (rhodamine phalloidin, red) and nuclei (DAPI, blue). (B). Cells were stained for ACE2 (anti-ACE2, green), MUC5AC+ goblet cells (anti-MUC5AC, cyan) F-actin (rhodamine phalloidin, red) and nuclei (DAPI, blue). (C). Cells were stained for ACE2 (anti-ACE2, green), MUC5B+ goblet cells (anti-MUC5B, cyan), F-actin (rhodamine phalloidin, red) and nuclei (DAPI, blue). Bar $=10 \mu \mathrm{m}$. See also Figures S1 and S2.

Figure 3. TMPRSS2 is expressed at higher levels in goblet cells. (A). The airway epithelia (described in Figure 1) were stained for cilia (anti-acetyl-alpha tubulin, cyan), TMPRSS2 (antiTMPRSS2, green), and F-actin (rhodamine phalloidin, red); the nuclei were stained with DAPI (blue). (B). The airway epithelia were stained for the identification of MUC5AC ${ }^{+}$goblet cells (anti-MUC5AC, cyan), TMPRSS2 (anti-TMPRSS2, green), F-actin (rhodamine phalloidin, red) and nuclei (DAPI, blue). (C). The airway epithelia were stained to identify MUC5B ${ }^{+}$goblet cells (anti-MUC5B, cyan), TMPRSS2 (anti-TMPRSS2, green), F-actin (rhodamine phalloidin, red) and nuclei (DAPI, blue). Bar $=10 \mu \mathrm{m}$.

Figure 4. SARS-CoV-2 infects goblet cells. (A). The airway epithelia were mock-infected or infected with SARS-CoV-2 at an MOI of 0.1. At 4 days post infection (DPI), the epithelia were fixed, permeabilized, and stained for the identification of cilia (anti-acetyl-alpha tubulin, cyan), SARS-CoV-2 N (anti-N, green), F-actin (rhodamine phalloidin, red) and nuclei (DAPI, blue). The arrows indicate SARS-CoV-2-infected nonciliated cells. (B). At 4 DPI, the epithelia (described in A) were stained for the goblet cell marker MUC5B (anti-MUC5B, cyan), SARS- 
CoV-2 N (anti-N, green), and F-actin (rhodamine phalloidin, red); the nuclei were stained with DAPI (blue). The arrows indicate SARS-CoV-2-infected MUC5B ${ }^{+}$goblet cells. (C). At 4 DPI, the epithelia (described in A) were embedded in paraffin, sectioned and stained for the detection of cilia (anti-acetyl-alpha tubulin, cyan) and SARS-CoV-2 N (anti-N, green); the nuclei were stained with DAPI (blue). The arrows indicate SARS-CoV-2-infected nonciliated cells. (D). At 4 DPI, the sectioned epithelia were stained for the goblet cell marker MUC5AC (anti-MUC5AC, cyan) and SARS-CoV-2 N (anti-N, green); the nuclei were also stained with DAPI (blue). The arrows indicate SARS-CoV-2-infected MUC5AC ${ }^{+}$goblet cells. (E). At 4 DPI, the sectioned epithelia were stained for the goblet cell marker MUC5B (anti-MUC5B, cyan) and SARS-CoV-2 $\mathrm{N}$ (anti-N, green); the nuclei were also stained with DAPI (blue). The arrows indicate SARSCoV-2-infected MUC5B ${ }^{+}$goblet cells. (A and B) Bar $=5 \mu \mathrm{m} .(\mathrm{C}, \mathrm{D}$ and $\mathrm{E}) \mathrm{Bar}=20 \mu \mathrm{m}$. See also Figures S3 and S4.

Figure 5. SARS-CoV-2 induces syncytia and cell sloughing. (A). The airway epithelia (mock or infected; described in Figure 4) were stained for SARS-CoV-2 N (anti-N, green) and F-actin (rhodamine phalloidin, red); the nuclei were also stained with DAPI (blue). The arrows indicate syncytia. Bar $=5 \mu \mathrm{m}$. (B). At $4 \mathrm{DPI}$, the sectioned epithelia were stained for the goblet cell marker MUC5AC (anti-MUC5AC, cyan) and SARS-CoV-2 N (anti-N, green), and the nuclei were also stained with DAPI (blue). The arrows indicate syncytia. Bar $=20 \mu \mathrm{m}$. (C). At 4 DPI, the airway epithelia were stained for SARS-CoV-2 N (anti-N, green) and F-actin (rhodamine phalloidin, red), and the nuclei were stained with DAPI (blue). The arrows indicate cell sloughing. Bar $=5 \mu \mathrm{m}$. (D). At 4 DPI, the sectioned epithelia were stained for cilia (anti-acetyl- 
alpha tubulin, cyan) and SARS-CoV-2 N (anti-N, green), and the nuclei were stained with DAPI (blue). The arrows indicate cell sloughing. Bar $=20 \mu \mathrm{m}$.

Figure 6. SARS-CoV-2 replicates at a higher rate and increases squamous metaplasia in the COPD epithelium. (A). The apical wash of the SARS-CoV-2-infected airway epithelia (described in Figure 4) was collected, and SARS-CoV-2 titration was performed based on the tissue culture infective dose $50\left(\mathrm{TCID}_{50}\right)$. The results from an independent experiment $(\mathrm{n}=3)$ are shown. The error bars show the SEMs. (B). The sectioned epithelia (described in Figure 4) were stained with Alcian blue. Bar $=50 \mu \mathrm{m}$. (C). At 4 DPI, the sectioned epithelia (COPD epithelia after mock or SARS-CoV-2 infection) were stained for the goblet cell marker MUC5B (antiMUC5B, cyan) and SARS-CoV-2 N (anti-N, green), and the nuclei were stained with DAPI (blue). Bar $=20 \mu \mathrm{m}$. (D). The height of the airway epithelia (described in C) was measured from the images $(n=5)$, the average of 12 points was plotted. See also Figure S5.

\section{Figure S1. ACE2 expression is higher in the COPD airway epithelium. (A) ACE2 mRNA} expression in total RNA extracted from the lung epithelial cell line (A549 cells) and primary NHBE cells from healthy adults and patients with COPD in monolayers or differentiated into airway epithelia was quantified by real-time PCR. The data were plotted as expression levels normalized to those in the NHBE healthy monolayer. The data were obtained by combining the quadruplet technical replicates of each sample. The graph represents the results from two independent real-time PCR runs. (B). ACE2 was detected in the airway epithelium (obtained after 4 weeks of differentiation of NHBE cells) by Western blotting. (C). The ACE2 signal (shown in B) was quantified (normalized to the alpha-tubulin level) and plotted relative to that in 
411 the healthy epithelium. (D). The apical sites of the airway epithelia were fixed and stained for

412 ACE2 (anti-ACE2, green), cilia (anti-acetyl-alpha tubulin, cyan) and F-actin (rhodamine

413 phalloidin, red). Deconvoluted Z-stack images are presented in the 3D view. (E). The apical sites

414 of the airway epithelia were fixed and stained for cilia (anti-acetyl-alpha tubulin, cyan), ACE2

415 (anti-ACE2, green), F-actin (rhodamine phalloidin, red) and nucleus (DAPI, blue). Bar $=15 \mu \mathrm{m}$.

416 (F). The airway epithelia (described in D) were stained for ACE2 (anti-ACE2, green), MUC5B

417 (anti-MUC5B, cyan) and F-actin (rhodamine phalloidin, red). Deconvoluted Z-stack images are

418 presented in the $3 \mathrm{D}$ view.

Figure S2. ACE2 expression within the goblet cell boundary. The airway epithelia (obtained after 4 weeks of differentiation of NHBE cells) were fixed and stained for ACE (anti-ACE2 antibody, green) and MUC5B (anti-MUC5B, cyan) or ZO-1 (anti-ZO-1 antibody, cyan) or ecadherin (anti-e-cadherin antibody, cyan). F-actin (red) and nuclei (blue) were stained with rhodamine phalloidin and DAPI, respectively. The images represent multiple random areas obtained from an independent experiment. Bar $=10 \mu \mathrm{m}$. infection (DPI), the epithelia were fixed, embedded in paraffin, sectioned, and stained for the basal cell marker P63 (anti-P63, green), SARS-CoV-2 spike (S) (anti-S, cyan), and F-actin

431 (rhodamine phalloidin, red); the nuclei were stained with DAPI (blue). Bar $=20 \mu \mathrm{m}$. 
Figure S4. SARS-CoV-2 does not infect basal cells in the airway epithelia. The airway

434 epithelia were mock-infected or infected with SARS-CoV-2 at an MOI of 0.1. At 4 days post

435 infection (DPI), the epithelia were fixed, permeabilized, and stained for cilia (anti-acetyl-alpha

436 tubulin, cyan), SARS-CoV-2 N (anti-N, green), and F-actin (rhodamine phalloidin, red); the

437 nuclei were stained with DAPI (blue). The images represent multiple independent random areas

438 from an independent experiment. Bar $=30 \mu \mathrm{m}$.

Figure S5. SARS-CoV-2 increases squamous metaplasia in the COPD airway epithelia. The

441 airway epithelia were mock-infected or infected with SARS-CoV-2 at an MOI of 0.1. At 4 days

442 post infection (DPI), the epithelia were fixed, permeabilized, and stained with hematoxylin and

$443 \quad \operatorname{eosin}(\mathrm{H} \& \mathrm{E}) . \mathrm{Bar}=50 \mu \mathrm{m}$.

\section{Graphical Abstract.}

446 Highlights:

1. The airway epithelium model recapitulates many bronchial pathological characteristics of

453 In Brief: 
Osan et al. showed that SARS-CoV-2 preferentially infects and replicates in nonciliated goblet cells inducing syncytium formation and cell sloughing. Our results suggest that goblet cells play a critical role in SARS-CoV-2-induced pathophysiology in the lung.

Acknowledgments. We thank the Microscopy Core (UND, Grand Forks) funded by NIH P20GM103442 of the INBRE program for providing access to an Olympus FV300 confocal microscope and Dr. Swojani Shrestha for the technical support provided. Histological services were provided by Histology Core (UND, Grand Forks) funded by NIH P20GM113123, NIH DaCCoTA CTR, NIH U54GM128729, and UNDSMHS funds. We also thank the Imaging Core (UND, Grand Forks) funded by NIH P20GM113123, NIH U54GM128729, and UNDSMHS funds for the IMARIS image analyses. We thank John Lee, Information Resources, UND, for preparing the graphical abstract. This work was funded by NIH/NIGMS P20GM113123 and in part by the Intramural Research Program of NIAID, NIH.

Author contributions. M.M., J.K.O., and S.N.T. conceived the project and designed the experiments. K.L.B. provided the primary cells as well as training and guidance on primary cell differentiation. M.M., J.K.O., and S.N.T. performed the experiments. S.N.T. generated the confocal images. F.F. and H.F. performed the virus infection experiments in the BSL-4 laboratory. J.K.O., B.A.D., and K.J. generated the IHC images, B.A.D. performed the H\&E and Alcian Blue staining, and M.M., J.K.O., and S.N.T. wrote the paper. M.M. and H.F. reviewed and edited the paper.

\section{STAR METHOD}


KEY RESOURCE TABLE

\begin{tabular}{|c|c|c|}
\hline REAGENT or RESOURSE & SOURCE & IDENTIFIER \\
\hline \multicolumn{3}{|l|}{ Antibodies } \\
\hline Rabbit monoclonal Anti-E-cadherin & $\begin{array}{l}\text { Cell Signaling } \\
\text { Technology }\end{array}$ & $\begin{array}{l}\text { Cat\#3195S; Clone 24E10; } \\
\text { RRID:AB_2291471 }\end{array}$ \\
\hline Rabbit monoclonal Anti-MUC5B & Atlas Antibodies & $\begin{array}{l}\text { Cat\# HPA008246; } \\
\text { RRID:AB_1854203 }\end{array}$ \\
\hline Mouse polyclonal Anti-MUC5AC & Abnova & $\begin{array}{l}\text { Cat\# H00004586-A01; } \\
\text { RRID:AB_606620 }\end{array}$ \\
\hline Rabbit monoclonal Anti-MUC5AC & $\begin{array}{l}\text { Cell Signaling } \\
\text { Technology }\end{array}$ & $\begin{array}{l}\text { Cat\#61193; Clone } \\
\text { E3O9I; } \\
\text { RRID: AB_2799603 }\end{array}$ \\
\hline Mouse monoclonal Anti-a-tubulin & Sigma-Aldrich & Cat\#T6199; RRID:AB_477583 \\
\hline Rabbit polyclonal Anti-ZO-1 & Thermo Fisher Scientific & Cat\#40-2200; RRID:AB_2533456 \\
\hline $\begin{array}{l}\text { Rabbit monoclonal Anti-Acetyl-a- } \\
\text { Tubulin }\end{array}$ & $\begin{array}{l}\text { Cell Signaling } \\
\text { Technology }\end{array}$ & Cat\#5335; RRID:AB_10544694 \\
\hline Mouse monoclonal Anti-TMPRSS2 & $\begin{array}{l}\text { Santa Cruz } \\
\text { Biotechnology }\end{array}$ & Cat\#sc-515727 \\
\hline Goat polyclonal Anti-ACE2 & R and D Systems & Cat\# AF933; RRID:AB_355722 \\
\hline Mouse monoclonal Anti-p63 & Abcam & $\begin{array}{l}\text { Cat\# ab735; Clone BC4A4; RRID: } \\
\text { AB_305870 }\end{array}$ \\
\hline Mouse monoclonal Anti-ACE2 & R and D Systems & $\begin{array}{l}\text { Cat\# MAB933; Clone } 171606 \\
\text { RRID:AB_2223153 }\end{array}$ \\
\hline $\begin{array}{l}\text { Mouse monoclonal Anti-SARS/SARS- } \\
\text { CoV-2 Nucleocapsid protein }\end{array}$ & Thermo Fisher Scientific & $\begin{array}{l}\text { Cat\# MA1-7404; Clone B46F } \\
\text { RRID:AB_1018422 }\end{array}$ \\
\hline $\begin{array}{l}\text { Rabbit polyclonal Anti-SARS/SARS- } \\
\text { CoV-2 Coronavirus Spike Protein }\end{array}$ & $\begin{array}{l}\text { Thermo Fisher } \\
\text { Scientific }\end{array}$ & $\begin{array}{l}\text { Cat\# PA5-81795; } \\
\text { RRID:AB_2788969 }\end{array}$ \\
\hline
\end{tabular}




\begin{tabular}{|c|c|c|}
\hline $\begin{array}{l}\text { Donkey Anti-Goat IgG antibody IRDye } \\
800 \mathrm{CW}\end{array}$ & LI-COR Biosciences & $\begin{array}{l}\text { Cat\# 926-32214, } \\
\text { RRID:AB_621846 }\end{array}$ \\
\hline $\begin{array}{l}\text { Goat Anti-Mouse IgG antibody IRDye } \\
\text { 680RD }\end{array}$ & LI-COR Biosciences & $\begin{array}{l}\text { Cat\#926- } \\
\text { 68070;RRID:AB_10956588 }\end{array}$ \\
\hline $\begin{array}{l}\text { Goat anti-Mouse IgG Antibody, Alexa } \\
\text { Fluor } 488\end{array}$ & Thermo Fisher Scientific & Cat\# A-11029; RRID:AB_2534088 \\
\hline $\begin{array}{l}\text { Goat anti-Rabbit IgG Antibody, Alexa } \\
\text { Fluor } 647\end{array}$ & Thermo Fisher Scientific & Cat\# A-21245; RRID:AB_2535813 \\
\hline \multicolumn{3}{|l|}{ Critical Commercial Assays } \\
\hline BCA protein assay kit & Thermo Fisher Scientific & 23225 \\
\hline AllPrep DNA/RNA Mini Kit & Qiagen & 80204 \\
\hline RNeasy Mini Kit & Qiagen & 74104 \\
\hline \multicolumn{3}{|l|}{ Virus strains } \\
\hline SARS CoV-2 WA 1 isolate & $\begin{array}{l}\text { Natalie J Thornburg, } \\
\text { CDC }\end{array}$ & GenBank: MN985325.1 \\
\hline \multicolumn{3}{|l|}{ Software } \\
\hline IMARIS 9.5.1 & Oxford Instruments & https://imaris.oxinst.com/ \\
\hline Fiji & ImageJ & https://imagej.net/Downloads \\
\hline Prism 8 & GraphPad & $\begin{array}{l}\text { https://www.graphpad.com/scientif } \\
\text { ic-software/prism/ }\end{array}$ \\
\hline $\begin{array}{l}\text { Sisson-Ammons Video Analysis } \\
\text { (SAVA) }\end{array}$ & Ammons Engineering & $\mathrm{N} / \mathrm{A}$ \\
\hline LASX & Leica Microsystem & $\mathrm{N} / \mathrm{A}$ \\
\hline Image Studio 5.2 & LI-COR Biosciences & $\begin{array}{l}\text { https://www.licor.com/bio/image- } \\
\text { studio/ }\end{array}$ \\
\hline Photoshop 2020 & Adobe & N/A \\
\hline Experimental models: & & \\
\hline
\end{tabular}




\begin{tabular}{|c|c|c|}
\hline Primary cells/ Cell lines & & \\
\hline $\begin{array}{l}\text { Normal Bronchial Epithelial Cells } \\
\text { (NHBE C16) }\end{array}$ & $\begin{array}{l}\text { Provided by Dr. Kristina } \\
\text { Bailey laboratory }\end{array}$ & \\
\hline COPD Bronchial Epithelial Cells & $\begin{array}{l}\text { Provided by Dr. Kristina } \\
\text { Bailey laboratory }\end{array}$ & \\
\hline A549 cells & $\begin{array}{l}\text { Provided by Dr. Peter } \\
\text { Collins laboratory }\end{array}$ & \\
\hline Vero cells & Heinz Feldmann, RML & \\
\hline $\begin{array}{l}\text { Chemical reagents, siRNA and } \\
\text { Media }\end{array}$ & & \\
\hline Airway Epithelial Cell Growth Medium & PromoCell & C21060 \\
\hline PneumaCult' ${ }^{\text {TM_ALI Medium }}$ & Stemcell Technologies & 05002 \\
\hline F-12 Medium & Thermo Fisher Scientific & 11765054 \\
\hline 2-mercaptoethanol & Sigma-Aldrich & M6250 \\
\hline Penicillin-Streptomycin & Thermo Fisher Scientific & 15140122 \\
\hline HyClone $^{\mathrm{TM}}$ Fetal Bovine Serum & Thermo Fisher Scientific & SH3007103 \\
\hline Amphotericin B & Thermo Fisher Scientific & 15290026 \\
\hline Goat Serum Blocking Solution & Vector Laboratories & S-1000-20 \\
\hline PureCol, Bovine Collagen & Cell Systems & 5005 \\
\hline $\begin{array}{l}\text { NucBlue TM fixed cell stain } \\
\text { ReadyProbes }\end{array}$ & Thermo Fisher Scientific & R37606 \\
\hline ProLong ${ }^{\mathrm{IM}}$ Gold anti-fade Mounting & Thermo Fisher Scientific & P36930 \\
\hline $\begin{array}{l}\text { Rhodamine Phalloidin (Amanita } \\
\text { phalloides) }\end{array}$ & Cytoskeleton Inc. & PHDR1 \\
\hline $16 \%$ Formaldehyde (Methanol free) & Polysciences & $18814-10$ \\
\hline $\begin{array}{l}\text { Intercept }{ }^{(P B S)} \text { Blocking Buffer and } \\
\text { Diluent Kit }\end{array}$ & LI-COR Biosciences & $927-76003$ \\
\hline
\end{tabular}


bioRxiv preprint doi: https://doi.org/10.1101/2020.11.11.379099; this version posted November 12,2020 . The copyright holder for this preprint (which was not certified by peer review) is the author/funder, who has granted bioRxiv a license to display the preprint in perpetuity. It is made available under aCC-BY-NC-ND 4.0 International license.

\begin{tabular}{|c|c|c|}
\hline TrypLETM Express Enzyme & Thermo Fisher Scientific & 12604021 \\
\hline \multicolumn{3}{|l|}{ Other } \\
\hline $\begin{array}{l}6.5 \mathrm{~mm} \text { Transwell® with } 0.4 \mu \mathrm{m} \text { Pore } \\
\text { Polyester Membrane Insert }\end{array}$ & Corning & 3470 \\
\hline Bolt $^{\mathrm{TM}} 4-12 \%$ Bis-Tris Plus Gels & Thermo Fisher Scientific & NW04120BOX \\
\hline iBlot $^{\mathrm{TM}} 2$ Transfer Stacks, PVDF & Thermo Fisher Scientific & IB24002 \\
\hline EVOM2 epithelial volt-ohmmeter & $\begin{array}{l}\text { World Precision } \\
\text { Instruments Inc }\end{array}$ & 177776 AB06J \\
\hline $\begin{array}{l}\text { FV3000 Confocal Laser Scanning } \\
\text { Microscope }\end{array}$ & Olympus Corporation & $\mathrm{N} / \mathrm{A}$ \\
\hline Leica DMi8 Inverted Microscope & Leica Microsystem & $\mathrm{N} / \mathrm{A}$ \\
\hline Odyssey® CLx Imaging System & LI-COR Biosciences & $\mathrm{N} / \mathrm{A}$ \\
\hline $\begin{array}{l}\text { Transmission Electron Microscope } \\
\text { HT7700 }\end{array}$ & Hitachi & $\mathrm{N} / \mathrm{A}$ \\
\hline CFX384 Real-Time PCR System & Bio-Rad & $\mathrm{N} / \mathrm{A}$ \\
\hline Epoch microplate spectrophotometer & BioTek & $\mathrm{N} / \mathrm{A}$ \\
\hline
\end{tabular}

RESOURCE AVIALABILITY

\section{Lead Contact}

480 Further information and requests for resources and reagents should be directed to and will be 481 fulfilled by the lead contact, Masfique Mehedi (masfique.mehedi@und.edu).

\section{Material Availability}

484 The materials and reagents generated in this study will be made available upon installment of a material transfer agreement (MTA). 
Competing interest. The authors declare no competing interests.

\section{EXPERIMENTAL MODEL AND SUBJECT DETAILS}

Cells. Primary normal human bronchial epithelial (NHBE) cells from either a deidentified healthy adult (female, aged 52 years, never-smoker) or a patient with chronic obstructive pulmonary disease (COPD) were obtained under an approved material transfer agreement (MTA) between the Mehedi Laboratory at University of North Dakota (UND) and Bailey Laboratory at University of Nebraska Medical Center (UNMC), Omaha, NE. A549 cells (a human lung epithelial cell line, ATCC-185) were obtained from Dr. Peter Collins at the National Institutes of Health $(\mathrm{NIH})$, Bethesda, MD, USA. Vero cells used for $\mathrm{TCID}_{50}$ assay were a resource of the Feldmann Lab at the Rocky Mountain Laboratories, Hamilton, MT.

Virus. The SARS-CoV-2 isolate nCoV-WA1-2020 (MN985325.1) was kindly provided by CDC as Vero passage 3 (Harcourt et al., 2020). The virus was propagated once in Vero E6 cells in DMEM (Sigma) supplemented with 2\% fetal bovine serum (Thermo Fisher Scientific), 1 mM Lglutamine (Thermo Fisher Scientific), $50 \mathrm{U} / \mathrm{ml}$ penicillin and $50 \mu \mathrm{g} / \mathrm{ml}$ streptomycin (Thermo Fisher Scientific) (virus isolation medium). The virus stock used in this study was $100 \%$ identical to the initially deposited GenBank sequence (MN985325.1); sequencing did not detect any virus stock contaminants.

Biosafety Statement. Work with SARS-CoV-2 was performed in the high biocontainment facilities at the Rocky Mountain Laboratories (RML), NAID, NIH in Hamilton, MT. All infectious work followed standard operating procedures (SOPs) approved by the Institutional Biosafety Committee. 


\section{METHOD DETAILS:}

511 Cell culture. We passaged the primary NHBE cells (passage 1) three times before differentiating them (passage 4) into a pseudostratified epithelium. For each passage, the cells were grown in a

513 100-mm culture dish (Corning Inc.) precoated with PurCol (Advanced Biometrics). The cells 514 were maintained in airway epithelial cell (AEC) growth medium (PromoCell) containing AEC 515 supplements (PromoCell), 2\% penicillin/streptomycin (Thermos Fisher Scientific), and 1\% 516 amphotericin B (Thermos Fisher Scientific) (complete AEC medium) at $37^{\circ} \mathrm{C}$ in an incubator 517 with $5 \% \mathrm{CO}_{2}$. The cells were grown to $90 \%$ confluency, and the medium was changed every 518 other day. Confluent monolayers of cells were dissociated with $5 \mathrm{ml}$ of TrypLE (Thermo Fisher

519 Scientific) and pelleted, and one-third of the cells were reseeded in a culture dish containing complete AEC medium for passaging. A549 cells were grown in F-12 medium (Thermo Fisher

521 Scientific) with 10\% HyClone fetal bovine serum (GE Healthcare), $2 \%$ penicillin/streptomycin, and $1 \%$ amphotericin $\mathrm{B}$.

Air-liquid interface (ALI) culture. Transwells $(6.5 \mathrm{~mm})$ with $0.4-\mu \mathrm{m}$-pore polyester membrane inserts (Corning Inc.) were coated with PureCol for 20 minutes before cell seeding. NHBE cells

$526\left(5 \times 10^{\wedge} 4\right)$ suspended in $200 \mu 1$ of complete AEC medium were seeded in the apical part of a 527 Transwell. Subsequently, $500 \mu 1$ of complete AEC medium was added to the basal part of the 528 Transwell. When the cells formed a confluent layer on the Transwell insert, the AEC medium 529 was removed from the apical part, and PneumaCult-ALI basal medium (Stemcell Technologies) 530 with the required supplements (Stemcell Technologies), 2\% penicillin/streptomycin and 1\% 531 amphotericin B (complete ALI basal medium) was added to the basal part. The ALI medium in 
532 the basal part was changed every other day, and the apical surface was washed with $1 \mathrm{x}$

533 Dulbecco's phosphate-buffered saline (DPBS) (Thermo Fisher Scientific) once per week initially

534 but more frequently when more mucus was observed on later days. However, the thickness of the

535 mucus was not determined. All the cells were differentiated for at least 4 weeks at $37^{\circ} \mathrm{C}$ in an

536 incubator with $5 \% \mathrm{CO}_{2}$.

Transportation of Transwells containing airway epithelia. The differentiated airway epithelia

for 22 days were transported in transportation medium at $4^{\circ} \mathrm{C}$ via overnight FedEx biological

540 material shipment to the Rocky Mountain Laboratories (RML, Hamilton, MT, USA). The

541 semisolid transportation medium was prepared by adding agarose to the complete AEC medium.

542 Upon receipt, the Transwells containing airway epithelia were immediately transferred into new

543 plates with complete AEC medium in the basal part and maintained before infection as described

544 in the section on ALI culture.

Virus infection. Virus infection was conducted in the high biocontainment facilities at RML,

547 NIAID, NIH. The 4-week-differentiated airway epithelia were washed with $200 \mu 1$ of $1 x$ PBS to

548 remove mucus and infected on the apical site with SARS-CoV-2 at an MOI of 0.1 in 1x PBS for

5491 hour (at $37^{\circ} \mathrm{C}$ with $5 \% \mathrm{CO}_{2}$ ). For mock infection, the assigned Transwells were similarly

550 incubated with 1x PBS without virus. The viral inoculum was then removed, and the epithelia

551 were washed twice with $200 \mu \mathrm{l}$ of 1x PBS. Two hundred microliters of apical wash and basal

552 medium were collected for virus titration. Fresh ALI medium (1000 $\mu 1)$ with supplements was

553 added to the basal part of each Transwell, and the apical part was kept empty. Mock-infected and 
SARS-CoV-2-infected Transwells were incubated for 4 days at $37^{\circ} \mathrm{C}$ in an incubator with $5 \%$ $\mathrm{CO}_{2}$.

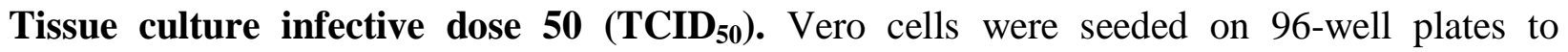
confluency, and SARS-CoV-2 endpoint titration was performed. The cells were inoculated with 10-fold dilutions of the supernatants collected during the experiment in DMEM, $2 \%$ FBS, Lglutamine and P/S. The cells were incubated for 7 days, and the cytopathic effect (CPE) was scored under a microscope. The $\mathrm{TCID}_{50}$ was calculated using the Reed-Muench formula (Reed and Muench, 1938).

Virus-infected sample collection. At 4 DPI, $200 \mu 1$ of 1x PBS was added to the apical site of the Transwell and incubated for several minutes, and the apical wash was collected for virus titration. Similarly, basal medium was collected for virus titration. All the samples were stored at $-80^{\circ} \mathrm{C}$ until titration. For PFA fixation, $200 \mu \mathrm{l}$ of $4 \%$ PFA was added at the apical site of the Transwells and incubated for 30 minutes, and the Transwells were further maintained overnight in 4\% PFA before being transported outside of the BSL4 lab according to the standard protocol of RML. For protein and RNA sample collections, $200 \mu \mathrm{l}$ of 1x PBS was added to the apical side of each Transwell, and cells were scrapped with a mini scrapper, collected into a cryovial, and

572 pelleted by being spun down with a table-top bench centrifuge. For the protein samples, $100 \mu 1$ 573 of $2 x$ SDS buffer was added to the cryovials containing the cell pellet, and the tube was boiled 574 for 10 minutes. After a quick spin, the samples were transferred into new tubes and removed from the BSL4 laboratory using a standard RML protocol. For the RNA samples, the cell pellets were resuspended in $600 \mu \mathrm{l}$ of RLT buffer (Qiagen) and transported out of the laboratory based 
on the RML protocol with $600 \mu$ l of ethanol. RNA was extracted using a Qiagen RNeasy mini kit (QIAGEN) and eluted in $50 \mu 1$ of RNase-free water. All the samples were shipped to Mehedi Lab at UND using an appropriate overnight biological shipment procedure.

\section{Hematoxylin and eosin (H\&E) staining. Four days post infection, NHBE-ALI and COPD-ALI} mock and infected Transwells were fixed with 4\% PFA for 24 hours. The membrane was cut off using a scalpel and embedded in paraffin mold. Five-micrometer sections were cut using a microtome. H\&E staining was performed by two 5-minute Histo-Clear incubations followed by three 5-minute incubations with 100\% ethanol, one 5-minute incubation with 95\% ethanol and one 5-minute incubation with $70 \%$ ethanol. Subsequently, the samples were washed with tap water for 2 minutes, incubated with $4 \%$ acidified Harries Hematoxylin for 2 minutes, washed with tap water for 2 minutes, and incubated with $0.5 \%$ lithium carbonate for 20 seconds. The samples were subsequently washed with tap water for 5 minutes, incubated with Eosin Y Solution with Pholoxine for 2 minutes and then subjected to a series of ethanol incubations: incubation with 70\% ethanol for 5 minutes, incubation with $95 \%$ ethanol for 5 minutes and three 5-minutes incubation with $100 \%$ ethanol. Finally, two 5-minute incubations with Histo-Clear were performed, and coverslips were then placed using permanent mounting medium.

Alcian blue staining. The 5- $\mu \mathrm{m}$ sectioned ALI-NHBE and COPD mock and infected samples were also subjected to Alcian blue staining. The sections were deparaffinized as described in the section on H\&E staining using Histo-Clear and ethanol. The cells were then washed with tap water for 2 minutes, incubated in 3\% acetic acid for 3 minutes and stained with Alcian blue for 30 minutes. The sections were subsequently rinsed with $3 \%$ acetic acid for 10 seconds to help 
600 prevent nonspecific staining. The sections were washed under tap water for 10 minutes and then

601 rinsed for 2 minutes in Milli-Q water. The sections were counterstained with nuclear fast red

602 solution for 5 minutes and washed under tap water for 5 minutes. The sections were subsequently

603 dehydrated and cleared with a gradient of alcohol and Histo-Clear as in the protocol used for

604 H\&E staining. The slides were mounted with permanent mounting medium and coverslipped.

605

606 Fluorescence imaging. The apical site of the airway epithelium was washed with PBS, and both

607 the apical and basal parts were fixed with 4\% paraformaldehyde (PFA) (Polysciences, Inc.), and

608 blocked with $10 \%$ goat serum (Vector Laboratories) solution in immunofluorescence (IF)

609 washing buffer (130 mM NaCl $2,7 \mathrm{mM} \mathrm{Na}_{2} \mathrm{HPO}_{4}, 3.5 \mathrm{mM} \mathrm{NaH}_{2} \mathrm{PO}_{4}, 7.7 \mathrm{mM} \mathrm{NaN}, 0.1 \%$ BSA,

$610 \quad 0.2 \%$ Triton-X 100 and $0.05 \%$ Tween-20) for 1 hour. The Transwell inserts were then incubated

611 with the following primary antibodies (Abs) (alone or in combination) in IF washing buffer

612 overnight at $4^{\circ} \mathrm{C}$ : anti-ZO-1 rabbit polyclonal (1:200) (Thermo Fisher Scientific), anti-E-

613 cadherin rabbit monoclonal (1:200) (Cell Signaling Technologies), anti-MUC5B rabbit

614 monoclonal (1:500) (Atlas Antibodies), anti-MUC5AC mouse polyclonal (1:100) (Abnova),

615 anti-MUC5AC rabbit monoclonal (1:200) (Cell Signaling Technology), anti-acetyl-alpha-tubulin

616 rabbit monoclonal (1:500) (Cell Signaling Technologies), anti-TMPRSS2 mouse monoclonal

617 (1:50) (Santa Cruz Biotechnology), anti-SARS CoV-2 nucleocapsid mouse monoclonal (1:10)

618 (Thermo Fisher Scientific) and anti-ACE2 mouse monoclonal (1:20) (R\&D Systems). The next

619 day, the inserts were washed with the IF washing buffer and incubated with an anti-mouse

620 AlexaFlour 488-conjugated Ab (1:200) (Thermo Fisher Scientific) and/or an anti-rabbit

621 AlexaFlour 647 (1:200) (Thermo Fisher Scientific) in IF washing buffer for 3 hours in the dark at

$6224{ }^{\circ} \mathrm{C}$. The cells were washed twice with PBS and incubated with rhodamine phalloidin (1:500) 
(Cytoskeleton Inc.) in IF washing buffer for 30 minutes at room temperature in the dark. After two washes with PBS, the cell nuclei were stained with NucBlue Fixed Cell Stain ReadyProbes (Thermo Fisher Scientific) for 30 minutes in the dark at room temperature. The epithelium was mounted on Tech-Med microscope slides (Thomas Scientific) using ProLong-Gold anti-fade mounting medium (Thermo Fisher Scientific). Images were captured using a confocal laserscanning microscope (Olympus FV3000) enabled with a 60X objective. The 405-nm laser was used to excite the DAPI signal for nucleus detection, the 488-nm laser was used to excite Alexa Flour 488 for MUC5AC, ACE2, TMPRSS2 or SARS CoV-2 nucleocapsid protein detection, the 561-nm laser was used to excite rhodamine phalloidin for F-actin detection, and the 640-nm laser was used to excite Alexa Flour 647 for MUC5B, acetyl-alpha-tubulin, E-cadherin, ZO-1 or MUC5AC detection. At least two random fields were selected per sample and imaged. The images were processed with Imaris software version 9.5.1 (Oxford Instruments Group) and used for the conversion of Z-stack images (.oir format) to .tiff format and for additional image postprocessing. Separately, the tiling of Z-stack (3x3) images was captured using a Leica DMi8 microscope followed by image processing using a 3D deconvolution image processing module in the LASX software (Leica Microsystem) associated with the microscope. We used nine independent images to quantify the total cell number based on F-actin (Texas Red channel) and goblet cells using the Alexa Flour 647 channel (anti-MUC5B) with the Fiji multipointer option. Similarly, we quantified the MUC5 $\mathrm{AC}^{+}$cells using at least two confocal images.

Immunohistochemistry: Healthy and COPD airway epithelia (mock or infected) were sectioned into 5- $\mu \mathrm{m}$ sections as described previously for immunohistochemistry. Before staining, the antigen retrieval process was performed using R-buffer A in a Retriever 2100. The slides were 
646 allowed to cool in the buffer overnight and washed three times in PBST (0.05\% Tween-20) for

64710 minutes. Using a Pap pen, a hydrophobic barrier was drawn around the tissue. Once the

648 hydrophobic barrier was dry, the tissue was incubated with blocking buffer (10\% goat serum in

649 PBS) for 2 hours in a humidified, light-protected chamber. After incubation with blocking buffer,

650 the tissue was immediately incubated with the following primary antibodies overnight at $4^{\circ} \mathrm{C}$ in a

651 humidified, light-protected chamber: SARS-CoV-2 N (1:50) (Thermo Fischer Scientific) for the

652 detection of SARS-CoV-2 nucleoprotein (N), SARS-CoV-2 spike (S) protein (1:100) (Thermo

653 Fischer Scientific) for the detection of SARS-CoV2 S protein, acetylated-alpha-tubulin (1:500)

654 (Cell Signaling Technologies) for the staining of ciliated cells, MUC5AC (1:500) (Cell Signaling

655 Technologies) and MUC5B (1:500) (Atlas Antibodies) for the staining of goblet cells, and P63

656 (1:100) (Abcam Inc.) for the staining of basal cells. The next day, the slides were washed three

657 times with PBST and then incubated for 45 minutes with anti-mouse Alexa Fluor 488 (Thermo

658 Fisher Scientific) and anti-rabbit Alexa Fluor 647 (Thermo Fisher Scientific) secondary

659 antibodies in a humidified, light-protected chamber. The slides were subsequently washed three

660 times with PBST, incubated with rhodamine phalloidin (1:100) (Cytoskeleton Inc.) for 30

661 minutes at room temperature in a humidified, light-protected chamber, and washed three times

662 with PBST. The nuclei were then stained by incubation with NucBlue Fixed Cell Stain

663 ReadyProbes (Thermo Fisher Scientific) for 5 minutes at room temperature in a humidified,

664 light-protected chamber. After incubation with the nuclear dye, the slides were washed once with

665 PBST and twice with DI water, and coverglass was then placed on the tissue section using

666 ProLong-Gold anti-fade mounting medium (Thermo Fisher Scientific). The slides were scanned

667 using a Leica DMi8 inverted fluorescence microscope with a 63X oil objective, and the images 
668

669

670

671

672

673

674

675

676

677

678

679

680

681

682

683

684

685

686

687

688

689

690

were further processed using a 3D deconvolution image processing module in the LASX software (Leica Microsystem) associated with the microscope.

Ciliary beat frequency (CBF). Cilia on the apical surface of the cells in the differentiated epithelial layer (after 4 weeks of differentiation) were visualized in the phase-contrast mode with a Leica DMi8 microscope with a 20X objective and an attached environment control chamber $\left(37^{\circ} \mathrm{C}\right.$ with $\left.5 \% \mathrm{CO}_{2}\right)$ (Leica Microsystems). For each Transwell, six different random fields were recorded for approximately 2.1 seconds at 120 frames per second. The images were captured at $37^{\circ} \mathrm{C}$ and analyzed using the Sisson-Ammons Video Analysis (SAVA) system V.2.1.15 to determine the $\mathrm{CBF}(\mathrm{Hz})$ (Ammons Engineering).

Transepithelial electrical resistance (TEER). The permeability of the differentiated epithelial layer (after 4 weeks of differentiation) was determined by measuring the TEER using an epithelial volt-ohm meter (EVOM2, World Precision Instruments, Inc.). The EVOM2 was calibrated according to the manufacturer's instructions, and the STX2 electrode was sterilized with $70 \%$ ethanol before use. The internal electrode (smaller in size) was placed in the apical part of each Transwell (PBS was added during the TEER reading), and the external electrode (larger in size) was placed in the basal part of the Transwell, which contained ALI basal medium, to measure the membrane voltage and resistance of the epithelial layer. An empty Transwell insert (filled with PBS) containing no NHBE cells was used to correct for the background resistance. Three readings were taken for each Transwell. The TEER value of each sample was calculated by subtracting the background value. 
Quantitative real-time PCR. Airway epithelia cultured on 6.5-mm Transwell membranes were washed and treated for 1 minute at RT with RLT buffer (Qiagen) with $1 \% \beta$-mercaptoethanol (Sigma-Aldrich). The cells were scraped using a cell scraper, collected into a QIAshredder tube and centrifuged at $15,000 \mathrm{rpm}$ and $4^{\circ} \mathrm{C}$ for 3 minutes. The eluate was used for the extraction of total RNA using a Total DNA/RNA Extraction Kit (Qiagen), and DNase I treatment was performed to remove DNA from the samples according to the manufacturer's instructions. We also followed a similar approach for the extraction of RNA from A549 cells. The RNA concentration was determined with an Epoch microplate spectrophotometer (BioTek). Five hundred nanograms of RNA was used for first-strand cDNA synthesis (Thermo Fisher Scientific) using Oligo(dT) primers (Thermo Fisher Scientific). qRT-PCR was performed using TaqMan assays (ACE2: Hs1085333_m1 and ACTB: Hs99999903_m1, for calibration) (Thermo Fisher Scientific) with the CFX384 Real-Time PCR System (Bio-Rad), and fold changes were calculated to determine the relative expression levels.

Western blotting. The airway epithelium cultured on $6.5-\mathrm{mm}$ Transwell membranes was washed with PBS, scraped out of all the cells, and pelleted by centrifugation at 10,000 rpm for 5 minutes. The cell pellet was incubated with 1x LDS loading buffer (Thermo Fisher Scientific) with proteinase inhibitor (Roche), transferred into a QIAshredder microcentrifuge tube, and centrifuged for 3 minutes at $15,000 \mathrm{rpm}$ in a tabletop centrifuge. The elusion from the QIAshredder was collected and stored in a freezer. The protein concentration was measured using a BCA protein assay kit (Thermo Fisher Scientific). For the detection of ACE2, total protein $(30 \mu \mathrm{g})$ was separated on $4-12 \%$ Bis-tris SDS polyacrylamide gels (reducing) and then subjected to dry blot transfer onto PVDF membranes according to the manufacturer's instructions 
714 (Life Technologies). The PVDF membrane was imaged using an Odyssey CLX system (Li-Cor

715 Biosciences). ACE2 was detected by Western blotting using anti-ACE2 goat polyclonal antibody

716 (R\&D Systems) and corresponding donkey anti-goat IRDye 800 secondary antibodies (Li-Cor

717 Biosciences). For the loading control, alpha-tubulin was detected by anti-alpha-tubulin mouse

718 monoclonal antibody (Sigma-Aldrich) and the corresponding goat anti-mouse IRDye 680

719 secondary antibody (Li-Cor Biosciences). Image Studio 5.2 (LI-COR Biosciences) was used to 720 quantify the protein signal.

Epithelial height measurement. Microscopic images of the IHC (described above) were used to quantify epithelial height by using the scale feature in LASX software of Leica DMi8

724 microscope. We used at least 4 independent slides for the measurement, at least three 725 independent reads per slide. The plastic membrane of the Transwell was not included in the 726 height measurement.

730 Statistical analysis: Parameters such as the number of replicates, number of independent 731 experiments, mean +/- SEM, and statistical significance are reported in the figures and figure 732 legends. A p-value less than 0.05 was considered to indicate significance. Where appropriate, the 733 statistical tests and post hoc statistical analysis methods are noted in the figure legends or 734 Methods section.

\section{References.}


Araya, J., Cambier, S., Markovics, J.A., Wolters, P., Jablons, D., Hill, A., Finkbeiner, W., Jones, K., Broaddus, V.C., Sheppard, D., et al. (2007). Squamous metaplasia amplifies pathologic epithelial-mesenchymal interactions in COPD patients. J Clin Invest 117, 3551-3562.

Barnes, P.J. (2013). New anti-inflammatory targets for chronic obstructive pulmonary disease. Nat Rev Drug Discov $12,543-559$.

Blanco-Melo, D., Nilsson-Payant, B.E., Liu, W.C., Uhl, S., Hoagland, D., Moller, R., Jordan, T.X., Oishi, K., Panis, M., Sachs, D., et al. (2020). Imbalanced Host Response to SARS-CoV-2 Drives Development of COVID-19. Cell. Borczuk, A.C., Salvatore, S.P., Seshan, S.V., Patel, S.S., Bussel, J.B., Mostyka, M., Elsoukkary, S., He, B., Del Vecchio, C., Fortarezza, F., et al. (2020). COVID-19 pulmonary pathology: a multi-institutional autopsy cohort from Italy and New York City. Mod Pathol.

Bradley, B.T., Maioli, H., Johnston, R., Chaudhry, I., Fink, S.L., Xu, H., Najafian, B., Deutsch, G., Lacy, J.M., Williams, T., et al. (2020). Histopathology and ultrastructural findings of fatal COVID-19 infections in Washington State: a case series. Lancet 396, 320-332.

Brusselle, G.G., Joos, G.F., and Bracke, K.R. (2011). New insights into the immunology of chronic obstructive pulmonary disease. Lancet $378,1015-1026$. CDC (2020). People with Certain Medical Conditions (CDC).

Coutard, B., Valle, C., de Lamballerie, X., Canard, B., Seidah, N.G., and Decroly, E. (2020). The spike glycoprotein of the new coronavirus 2019-nCoV contains a furin-like cleavage site absent in $\mathrm{CoV}$ of the same clade. Antiviral Res 176, 104742.

Crystal, R.G. (2014). Airway basal cells. The "smoking gun" of chronic obstructive pulmonary disease. Am J Respir Crit Care Med 190, 1355-1362.

Dhand, R., and Li, J. (2020). Coughs and Sneezes: Their Role in Transmission of Respiratory Viral Infections, Including SARS-CoV-2. Am J Respir Crit Care Med 202, 651-659.

Falasca, L., Nardacci, R., Colombo, D., Lalle, E., Di Caro, A., Nicastri, E., Antinori, A., Petrosillo, N., Marchioni, L., Biava, G., et al. (2020). Post-Mortem Findings in Italian Patients with COVID-19 - a Descriptive Full Autopsy Study of cases with and without co-morbidities. J Infect Dis.

Franks, T.J., Chong, P.Y., Chui, P., Galvin, J.R., Lourens, R.M., Reid, A.H., Selbs, E., McEvoy, C.P., Hayden, C.D., Fukuoka, J., et al. (2003). Lung pathology of severe acute respiratory syndrome (SARS): a study of 8 autopsy cases from Singapore. Hum Pathol 34, 743-748.

Fulcher, M.L., Gabriel, S., Burns, K.A., Yankaskas, J.R., and Randell, S.H. (2005). Well-differentiated human airway epithelial cell cultures. Methods Mol Med 107, 183-206.

Giacca, M., Bussani, R., Schneider, E., Zentilin, L., Collesi, C., Ali, H., Braga, L., Secco, I., Volpe, M.C., and Colliva, A. (2020). Persistence of viral RNA, widespread thrombosis and abnormal cellular syncytia are hallmarks of COVID-19 lung pathology. medRxiv.

Gohy, S., Carlier, F.M., Fregimilicka, C., Detry, B., Lecocq, M., Ladjemi, M.Z., Verleden, S., Hoton, D., Weynand, B., Bouzin, C., et al. (2019). Altered generation of ciliated cells in chronic obstructive pulmonary disease. Sci Rep 9 , 17963.

Guan, W.J., Ni, Z.Y., Hu, Y., Liang, W.H., Ou, C.Q., He, J.X., Liu, L., Shan, H., Lei, C.L., Hui, D.S.C., et al. (2020). Clinical Characteristics of Coronavirus Disease 2019 in China. N Engl J Med 382, 1708-1720. Hamming, I., Timens, W., Bulthuis, M.L., Lely, A.T., Navis, G., and van Goor, H. (2004). Tissue distribution of ACE2 protein, the functional receptor for SARS coronavirus. A first step in understanding SARS pathogenesis. J Pathol 203, 631-637.

Hao, S., Ning, K., Kuz, C.A., Vorhies, K., Yan, Z., and Qiu, J. (2020). Long-Term Modeling of SARS-CoV-2 Infection of In Vitro Cultured Polarized Human Airway Epithelium. mBio 11.

Harcourt, J., Tamin, A., Lu, X., Kamili, S., Sakthivel, S.K., Murray, J., Queen, K., Tao, Y., Paden, C.R., Zhang, J., et al. (2020). Severe Acute Respiratory Syndrome Coronavirus 2 from Patient with Coronavirus Disease, United States. Emerg Infect Dis 26, 1266-1273.

Higham, A., Quinn, A.M., Cancado, J.E.D., and Singh, D. (2019). The pathology of small airways disease in COPD: historical aspects and future directions. Respir Res 20, 49.

Hoffmann, M., Kleine-Weber, H., Schroeder, S., Kruger, N., Herrler, T., Erichsen, S., Schiergens, T.S., Herrler, G., Wu, N.H., Nitsche, A., et al. (2020). SARS-CoV-2 Cell Entry Depends on ACE2 and TMPRSS2 and Is Blocked by a Clinically Proven Protease Inhibitor. Cell 181, 271-280 e278.

Hou, Y.J., Okuda, K., Edwards, C.E., Martinez, D.R., Asakura, T., Dinnon, K.H., 3rd, Kato, T., Lee, R.E., Yount, B.L., Mascenik, T.M., et al. (2020). SARS-CoV-2 Reverse Genetics Reveals a Variable Infection Gradient in the Respiratory Tract. Cell 182, 429-446 e414. 
Huang, C., Wang, Y., Li, X., Ren, L., Zhao, J., Hu, Y., Zhang, L., Fan, G., Xu, J., Gu, X., et al. (2020). Clinical features of patients infected with 2019 novel coronavirus in Wuhan, China. Lancet 395, 497-506.

Jia, H.P., Look, D.C., Hickey, M., Shi, L., Pewe, L., Netland, J., Farzan, M., Wohlford-Lenane, C., Perlman, S., and McCray, P.B., Jr. (2006). Infection of human airway epithelia by SARS coronavirus is associated with ACE2 expression and localization. Adv Exp Med Biol 581, 479-484.

Jia, H.P., Look, D.C., Shi, L., Hickey, M., Pewe, L., Netland, J., Farzan, M., Wohlford-Lenane, C., Perlman, S., and McCray, P.B., Jr. (2005). ACE2 receptor expression and severe acute respiratory syndrome coronavirus infection depend on differentiation of human airway epithelia. J Virol 79, 14614-14621.

Kim, V., Oros, M., Durra, H., Kelsen, S., Aksoy, M., Cornwell, W.D., Rogers, T.J., and Criner, G.J. (2015). Chronic bronchitis and current smoking are associated with more goblet cells in moderate to severe COPD and smokers without airflow obstruction. PLoS One 10, e0116108.

Kiyokawa, H., and Morimoto, M. (2020). Notch signaling in the mammalian respiratory system, specifically the trachea and lungs, in development, homeostasis, regeneration, and disease. Dev Growth Differ 62, 67-79. Lamers, M.M., Beumer, J., van der Vaart, J., Knoops, K., Puschhof, J., Breugem, T.I., Ravelli, R.B.G., Paul van Schayck, J., Mykytyn, A.Z., Duimel, H.Q., et al. (2020). SARS-CoV-2 productively infects human gut enterocytes. Science 369, 50-54.

Leung, J.M., Niikura, M., Yang, C.W.T., and Sin, D.D. (2020). COVID-19 and COPD. Eur Respir J 56. Li, M.Y., Li, L., Zhang, Y., and Wang, X.S. (2020). Expression of the SARS-CoV-2 cell receptor gene ACE2 in a wide variety of human tissues. Infect Dis Poverty 9, 45.

Li, W., Moore, M.J., Vasilieva, N., Sui, J., Wong, S.K., Berne, M.A., Somasundaran, M., Sullivan, J.L., Luzuriaga, K., Greenough, T.C., et al. (2003). Angiotensin-converting enzyme 2 is a functional receptor for the SARS coronavirus. Nature 426, 450-454.

Lippi, G., and Henry, B.M. (2020). Chronic obstructive pulmonary disease is associated with severe coronavirus disease 2019 (COVID-19). Respir Med 167, 105941.

Lukassen, S., Chua, R.L., Trefzer, T., Kahn, N.C., Schneider, M.A., Muley, T., Winter, H., Meister, M., Veith, C., Boots, A.W., et al. (2020). SARS-CoV-2 receptor ACE2 and TMPRSS2 are primarily expressed in bronchial transient secretory cells. EMBO J 39, e105114.

Martines, R.B., Ritter, J.M., Matkovic, E., Gary, J., Bollweg, B.C., Bullock, H., Goldsmith, C.S., Silva-Flannery, L., Seixas, J.N., Reagan-Steiner, S., et al. (2020). Pathology and Pathogenesis of SARS-CoV-2 Associated with Fatal Coronavirus Disease, United States. Emerg Infect Dis 26, 2005-2015.

Matrosovich, M.N., Matrosovich, T.Y., Gray, T., Roberts, N.A., and Klenk, H.D. (2004). Human and avian influenza viruses target different cell types in cultures of human airway epithelium. Proc Natl Acad Sci U S A 101, 4620-4624.

Okuda, K., Chen, G., Subramani, D.B., Wolf, M., Gilmore, R.C., Kato, T., Radicioni, G., Kesimer, M., Chua, M., Dang, H., et al. (2019). Localization of Secretory Mucins MUC5AC and MUC5B in Normal/Healthy Human Airways. Am J Respir Crit Care Med 199, 715-727.

Pawlina, W. (2016). In Histology a Text Book and Atlas with Correlated Cell and Molecualr Biology, W. Pawlina, ed. (Wolters Kluwer), pp. 671-697.

Persson, B.D., Jaffe, A.B., Fearns, R., and Danahay, H. (2014). Respiratory syncytial virus can infect basal cells and alter human airway epithelial differentiation. PLoS One 9, e102368.

Polosukhin, V.V., Cates, J.M., Lawson, W.E., Zaynagetdinov, R., Milstone, A.P., Massion, P.P., Ocak, S., Ware, L.B., Lee, J.W., Bowler, R.P., et al. (2011). Bronchial secretory immunoglobulin a deficiency correlates with airway inflammation and progression of chronic obstructive pulmonary disease. Am J Respir Crit Care Med 184, 317-327. Qi, F., Qian, S., Zhang, S., and Zhang, Z. (2020). Single cell RNA sequencing of 13 human tissues identify cell types and receptors of human coronaviruses. Biochem Biophys Res Commun 526, 135-140.

Randell, S.H. (2006). Airway epithelial stem cells and the pathophysiology of chronic obstructive pulmonary disease. Proc Am Thorac Soc 3, 718-725.

Rayner, R.E., Makena, P., Prasad, G.L., and Cormet-Boyaka, E. (2019). Optimization of Normal Human Bronchial Epithelial (NHBE) Cell 3D Cultures for in vitro Lung Model Studies. Sci Rep 9, 500.

Reed, L.J., and Muench, H. (1938). A simple method of estimating fifty per cent endpoints. American journal of epidemiology 27, 493-497.

Reid, A.T., Veerati, P.C., Gosens, R., Bartlett, N.W., Wark, P.A., Grainge, C.L., Stick, S.M., Kicic, A., Moheimani, F., Hansbro, P.M., et al. (2018). Persistent induction of goblet cell differentiation in the airways: Therapeutic approaches. Pharmacol Ther 185, 155-169. 
Rigden, H.M., Alias, A., Havelock, T., O'Donnell, R., Djukanovic, R., Davies, D.E., and Wilson, S.J. (2016). Squamous Metaplasia Is Increased in the Bronchial Epithelium of Smokers with Chronic Obstructive Pulmonary Disease. PLoS One 11, e0156009.

Rogers, D.F. (2003). The airway goblet cell. Int J Biochem Cell Biol 35, 1-6.

Schaefer, I.M., Padera, R.F., Solomon, I.H., Kanjilal, S., Hammer, M.M., Hornick, J.L., and Sholl, L.M. (2020). In situ detection of SARS-CoV-2 in lungs and airways of patients with COVID-19. Mod Pathol 33, 2104-2114.

Shaykhiev, R. (2019). Emerging biology of persistent mucous cell hyperplasia in COPD. Thorax 74, 4-6. Shimura, S., Andoh, Y., Haraguchi, M., and Shirato, K. (1996). Continuity of airway goblet cells and intraluminal mucus in the airways of patients with bronchial asthma. Eur Respir J 9, 1395-1401.

Shulla, A., Heald-Sargent, T., Subramanya, G., Zhao, J., Perlman, S., and Gallagher, T. (2011). A transmembrane serine protease is linked to the severe acute respiratory syndrome coronavirus receptor and activates virus entry. J Virol 85, 873-882.

Sims, A.C., Baric, R.S., Yount, B., Burkett, S.E., Collins, P.L., and Pickles, R.J. (2005). Severe acute respiratory syndrome coronavirus infection of human ciliated airway epithelia: role of ciliated cells in viral spread in the conducting airways of the lungs. J Virol 79, 15511-15524.

Sin, D.D. (2020). COVID-19 in COPD: A growing concern. EClinicalMedicine 26, 100546.

Sungnak, W., Huang, N., Becavin, C., Berg, M., Queen, R., Litvinukova, M., Talavera-Lopez, C., Maatz, H., Reichart, D., Sampaziotis, F., et al. (2020). SARS-CoV-2 entry factors are highly expressed in nasal epithelial cells together with innate immune genes. Nat Med 26, 681-687.

To, K.F., and Lo, A.W. (2004). Exploring the pathogenesis of severe acute respiratory syndrome (SARS): the tissue distribution of the coronavirus (SARS-CoV) and its putative receptor, angiotensin-converting enzyme 2 (ACE2). J Pathol 203, 740-743.

Wang, B.Y., Gil, J., Kaufman, D., Gan, L., Kohtz, D.S., and Burstein, D.E. (2002). P63 in pulmonary epithelium, pulmonary squamous neoplasms, and other pulmonary tumors. Hum Pathol 33, 921-926.

Wang, W., Xu, Y., Gao, R., Lu, R., Han, K., Wu, G., and Tan, W. (2020). Detection of SARS-CoV-2 in Different Types of Clinical Specimens. JAMA.

Whitsett, J.A. (2018). Airway Epithelial Differentiation and Mucociliary Clearance. Ann Am Thorac Soc 15, S143S148.

Wolf, S., Perez, G.F., Mukharesh, L., Isaza, N., Preciado, D., Freishtat, R.J., Pillai, D., Rose, M.C., and Nino, G. (2017). Conditional reprogramming of pediatric airway epithelial cells: A new human model to investigate early-life respiratory disorders. Pediatr Allergy Immunol 28, 810-817.

Wolfel, R., Corman, V.M., Guggemos, W., Seilmaier, M., Zange, S., Muller, M.A., Niemeyer, D., Jones, T.C., Vollmar, P., Rothe, C., et al. (2020). Virological assessment of hospitalized patients with COVID-2019. Nature 581, 465-469.

Yang, X., Yu, Y., Xu, J., Shu, H., Xia, J., Liu, H., Wu, Y., Zhang, L., Yu, Z., Fang, M., et al. (2020). Clinical course and outcomes of critically ill patients with SARS-CoV-2 pneumonia in Wuhan, China: a single-centered, retrospective, observational study. Lancet Respir Med 8, 475-481.

Zhang, H., Rostami, M.R., Leopold, P.L., Mezey, J.G., O'Beirne, S.L., Strulovici-Barel, Y., and Crystal, R.G. (2020). Expression of the SARS-CoV-2 ACE2 Receptor in the Human Airway Epithelium. Am J Respir Crit Care Med 202, 219-229.

Zhang, L., Peeples, M.E., Boucher, R.C., Collins, P.L., and Pickles, R.J. (2002). Respiratory syncytial virus infection of human airway epithelial cells is polarized, specific to ciliated cells, and without obvious cytopathology. J Virol 76, 5654-5666.

Zhao, Q., Meng, M., Kumar, R., Wu, Y., Huang, J., Lian, N., Deng, Y., and Lin, S. (2020). The impact of COPD and smoking history on the severity of COVID-19: A systemic review and meta-analysis. J Med Virol. Ziegler, C.G.K., Allon, S.J., Nyquist, S.K., Mbano, I.M., Miao, V.N., Tzouanas, C.N., Cao, Y., Yousif, A.S., Bals, J., Hauser, B.M., et al. (2020). SARS-CoV-2 Receptor ACE2 Is an Interferon-Stimulated Gene in Human Airway Epithelial Cells and Is Detected in Specific Cell Subsets across Tissues. Cell. 
A Pseudostarified

Healthy epithellium

B Pseudostartitied

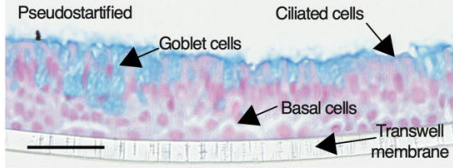

Healthy epithellium

G
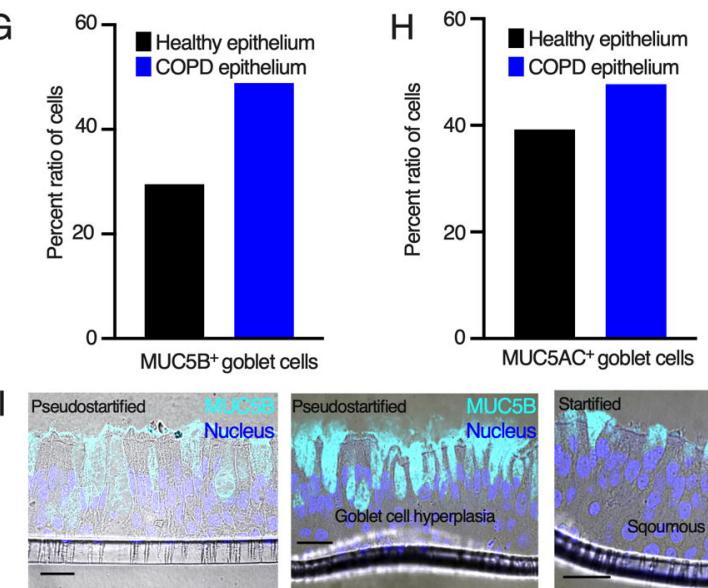

Healthy epithellium

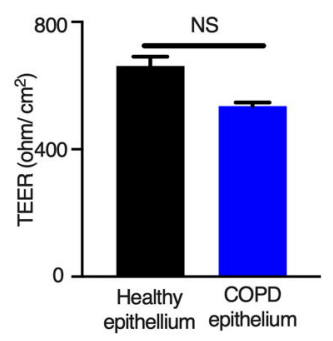

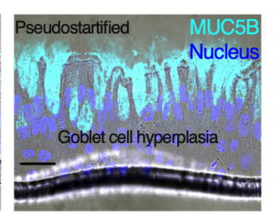

COPD epithelium

$\mathrm{K}$

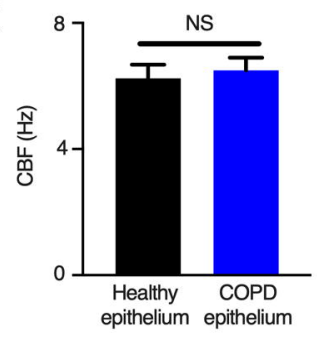

Pseudostartified

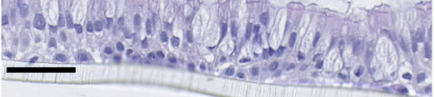

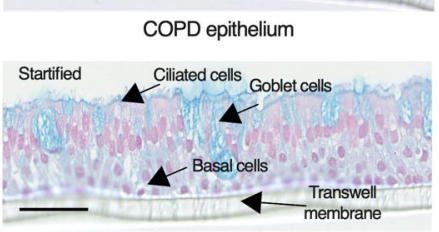

COPD epithelium

C Healthy epithelium COPD epithelium
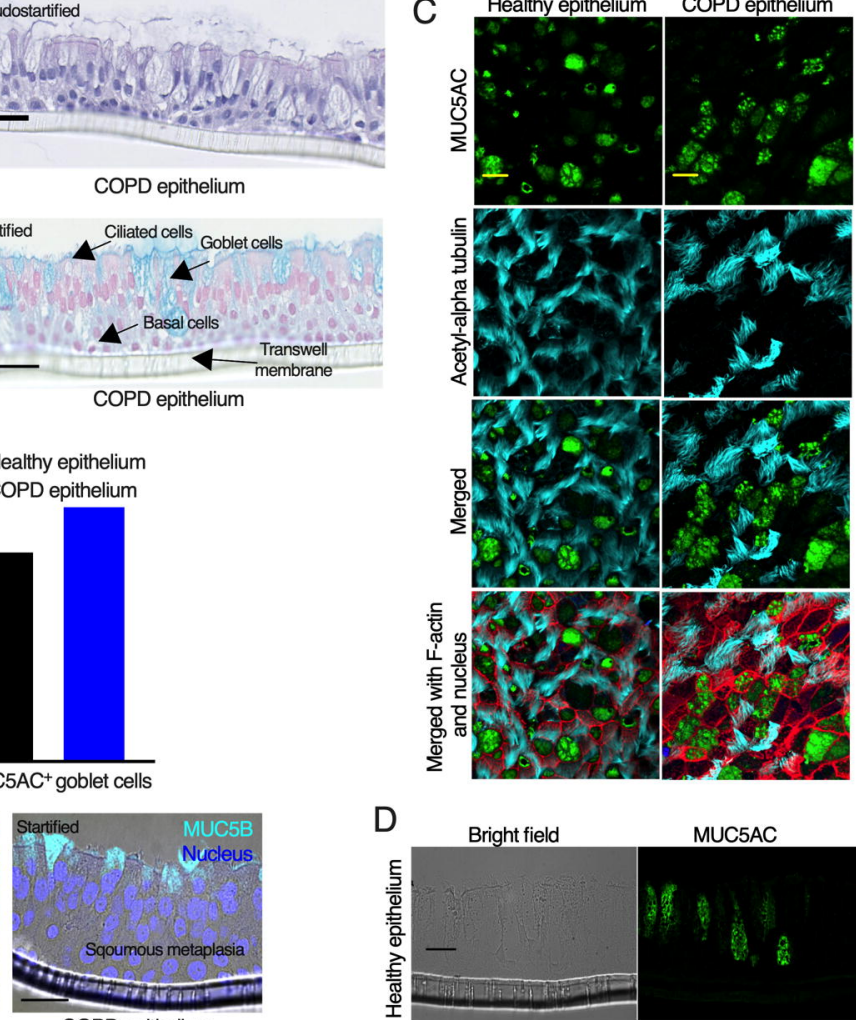

COPD epithelium
D

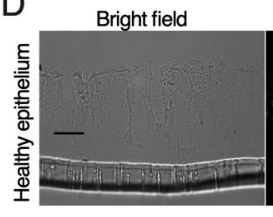

$\mathrm{F}$

F
MUC5AC

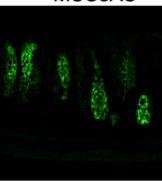

Acetyl-alpha tubulin

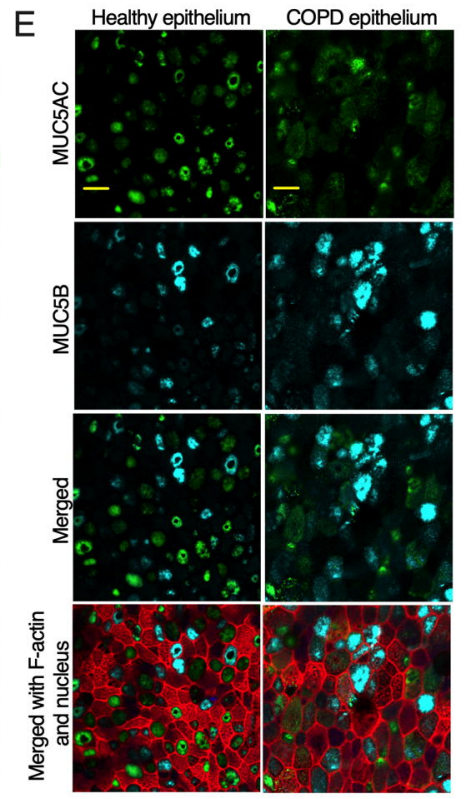

Mergd with F-actin and nucleus

\section{Figure 1}




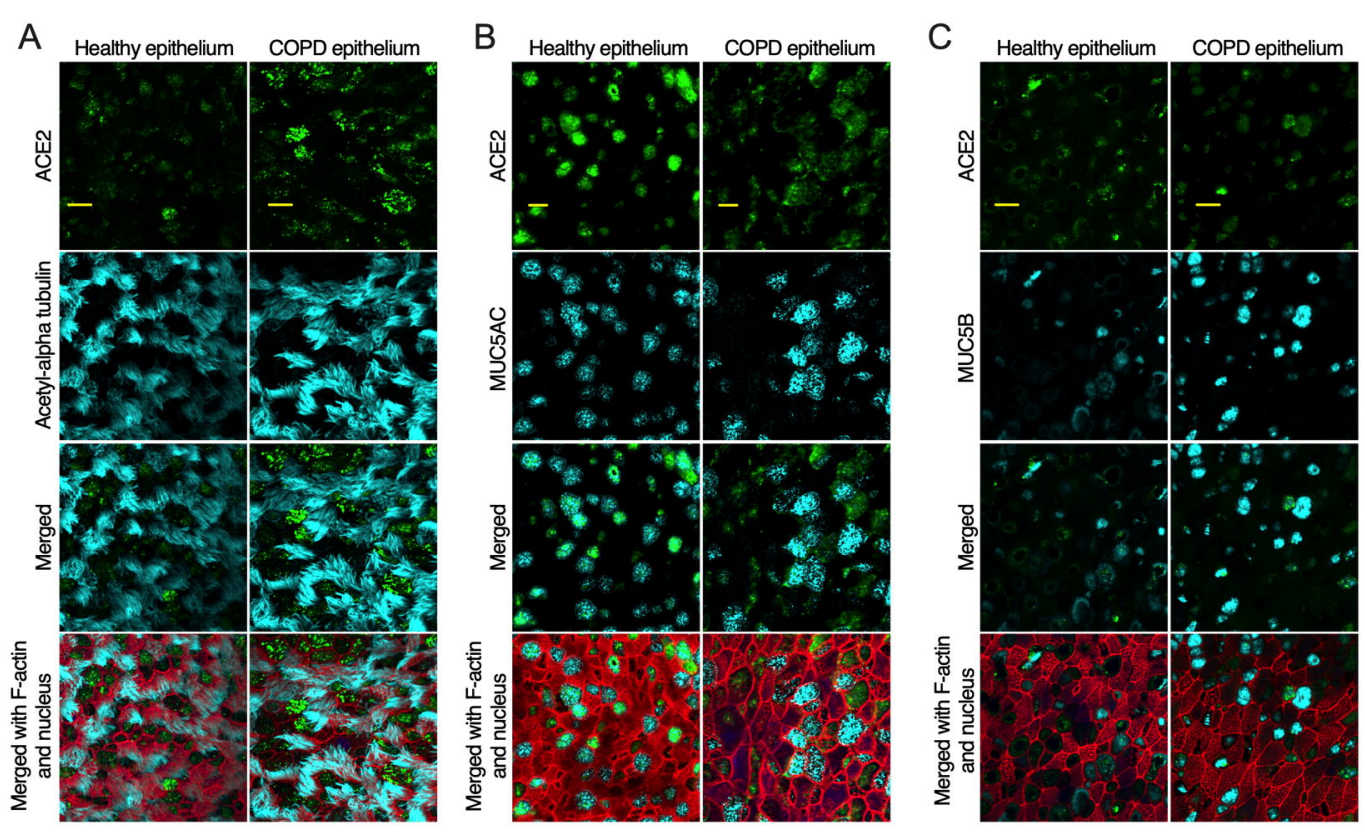

Figure 2 
A Acetyl-alpha tubulin

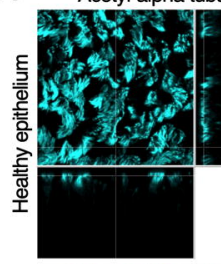

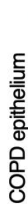

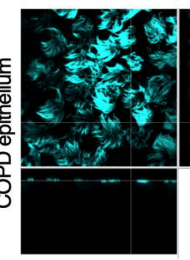

B

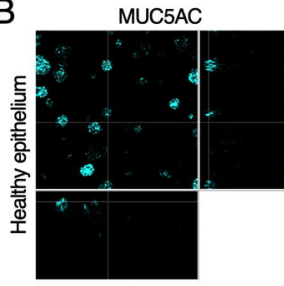

E
$\frac{.}{0}$
$\frac{0}{0}$
0
0
0
0
0

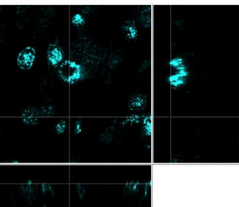

C

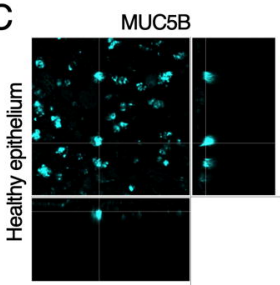

8

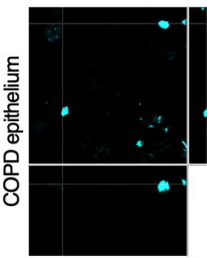

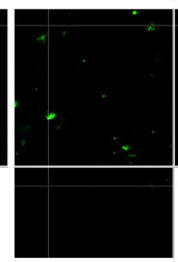

TMPRSS2
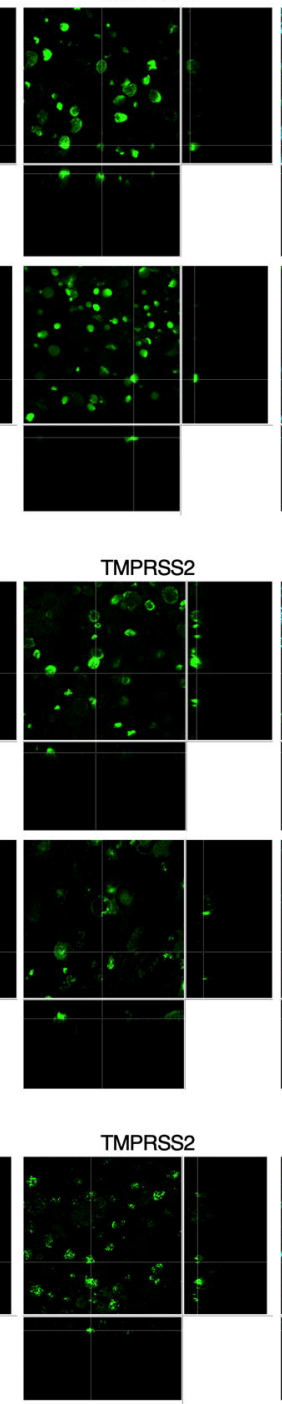

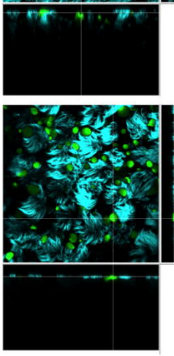

Merged
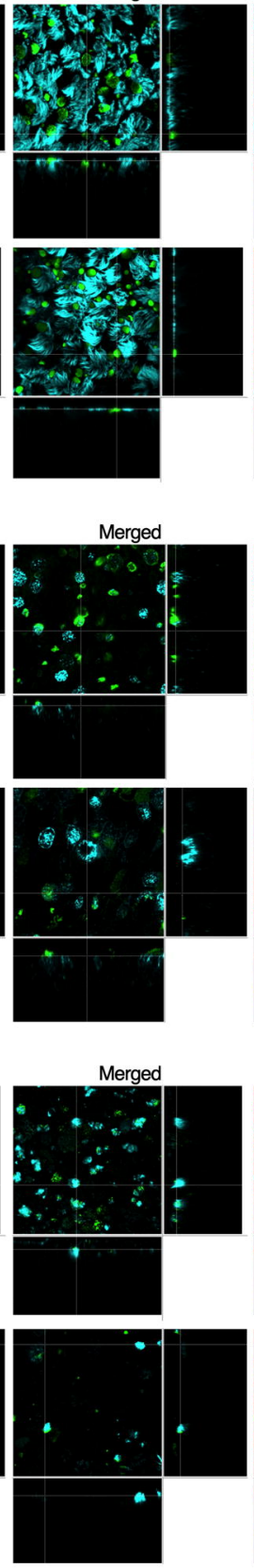

Merged with F-actin and nucleus
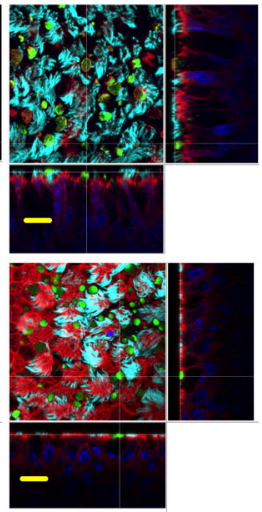

Merged with F-actin and nucleus

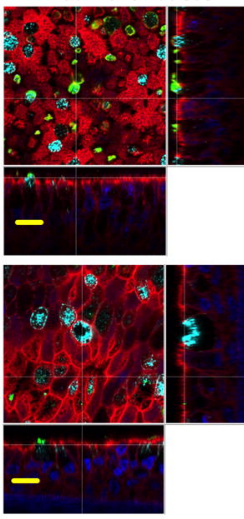

Merged with

F-actin and nucleus
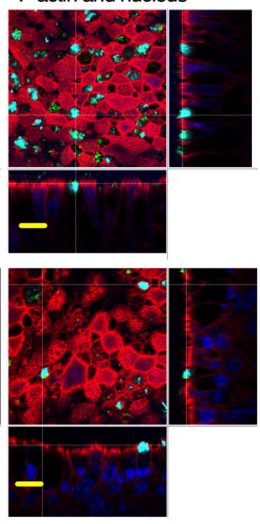

Figure 3 


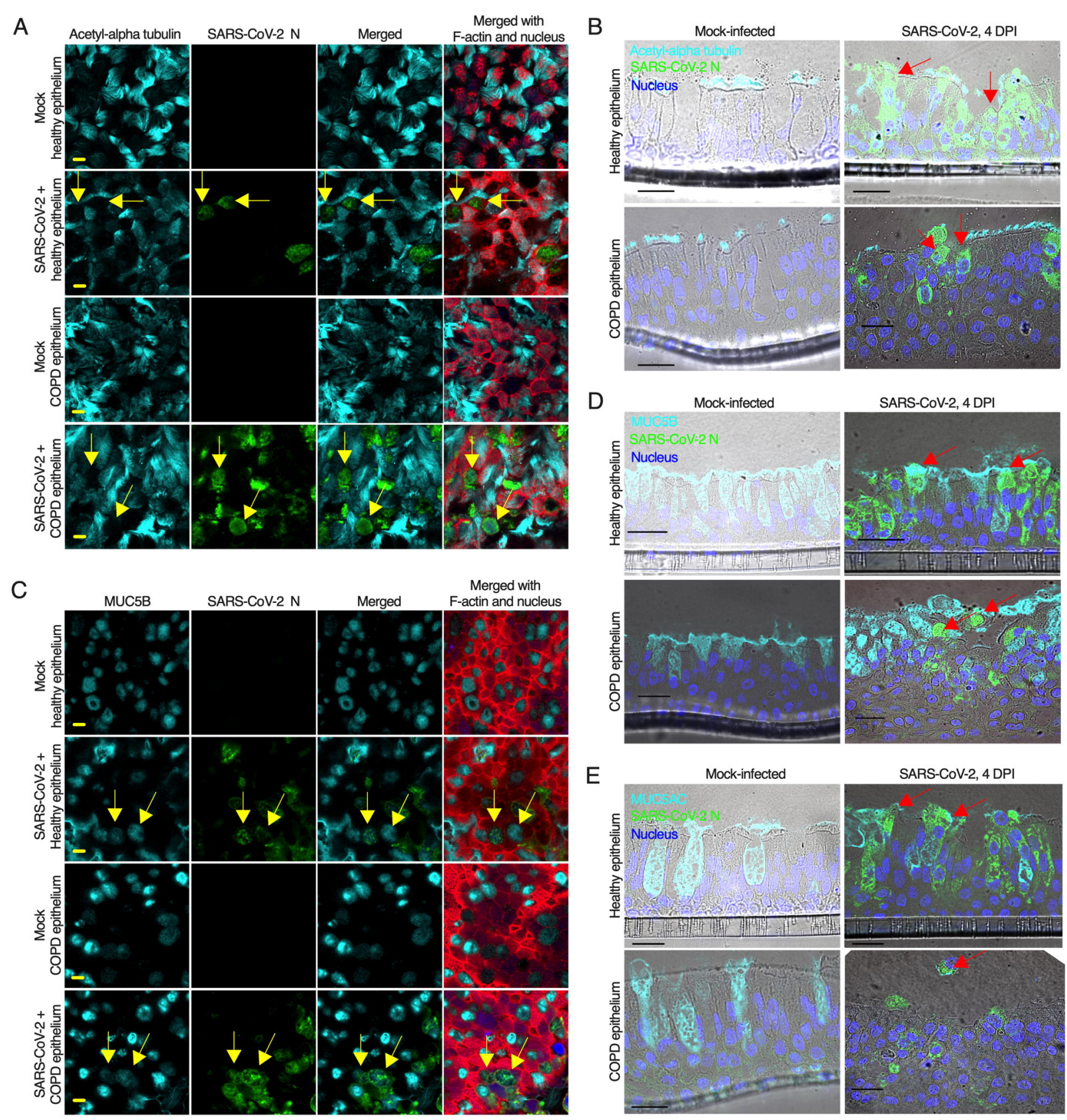

Figure 4 

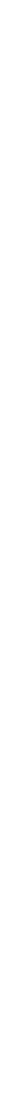

D

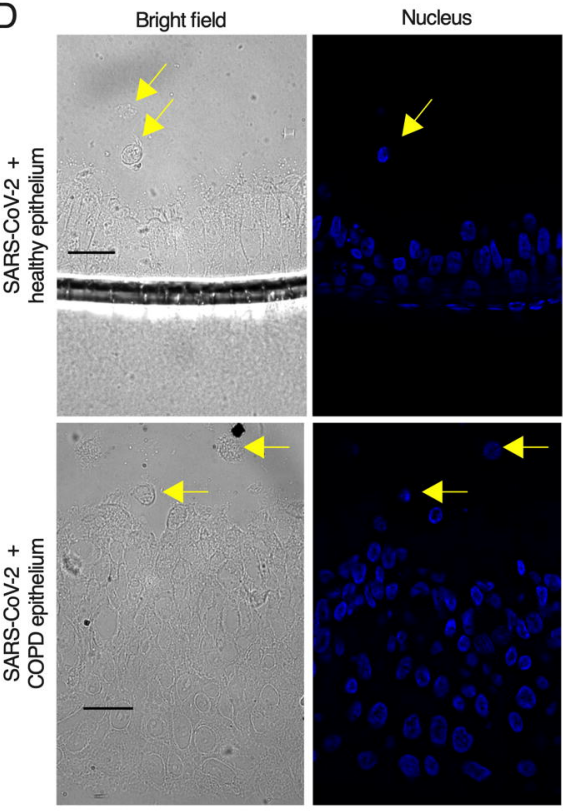

Acetyl-alpha tubulin

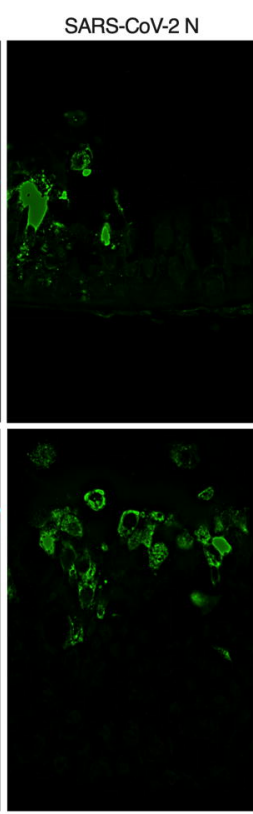

Merged with F-actin
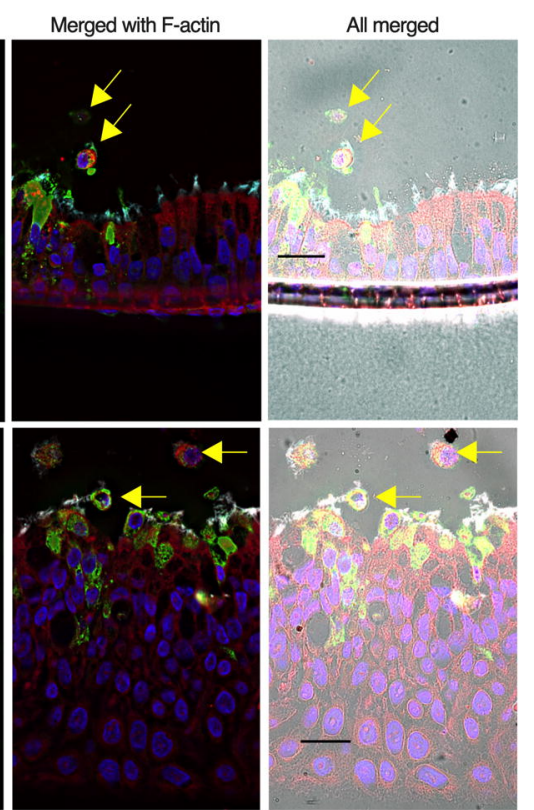

Figure 5 


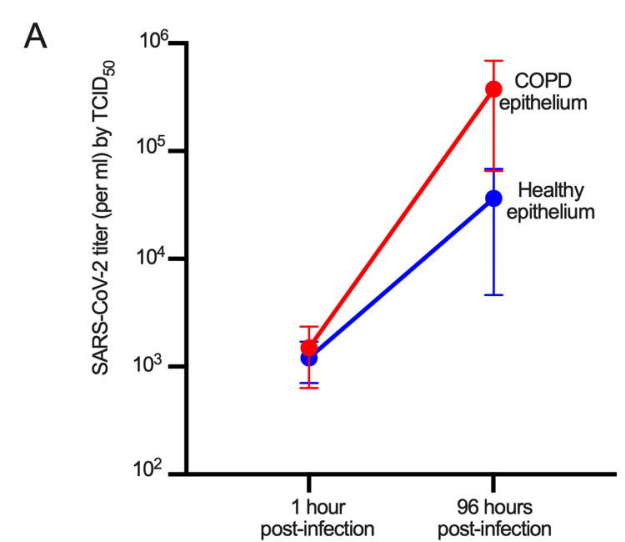

B

Mock-infected
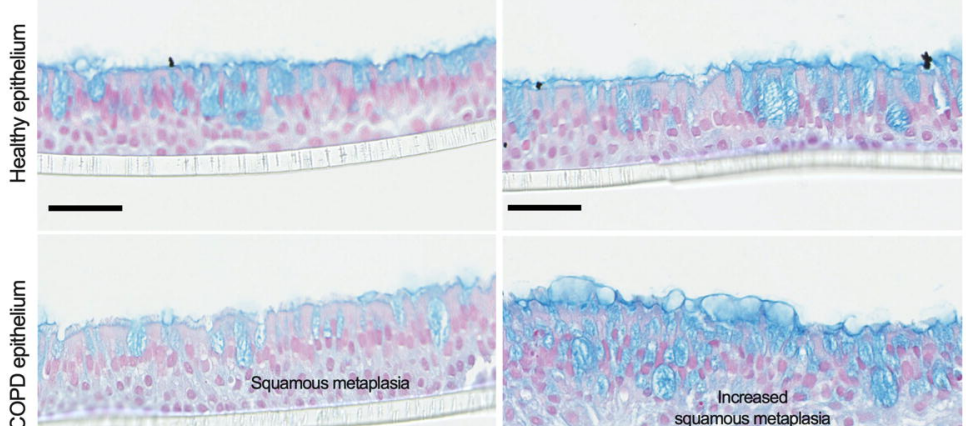

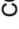

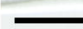

MUC5B

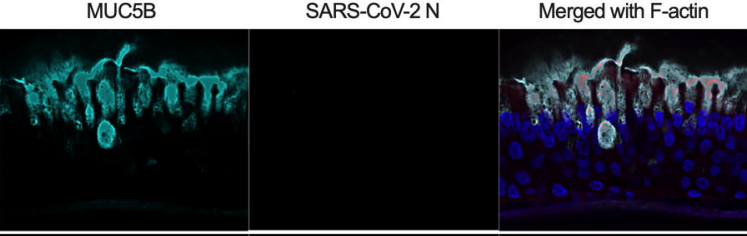

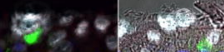
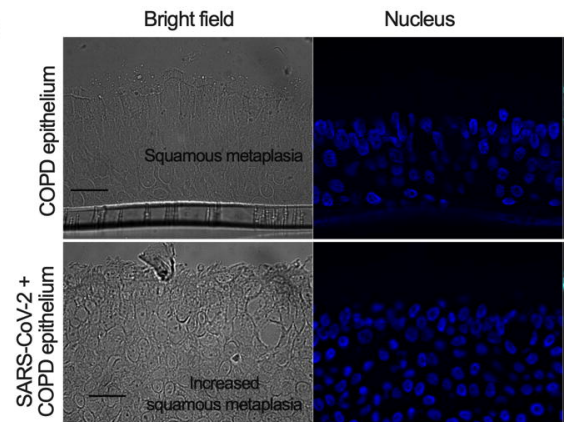

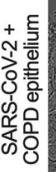

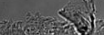

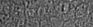

1.6
Increased
squamous mela

squamous metaplasia
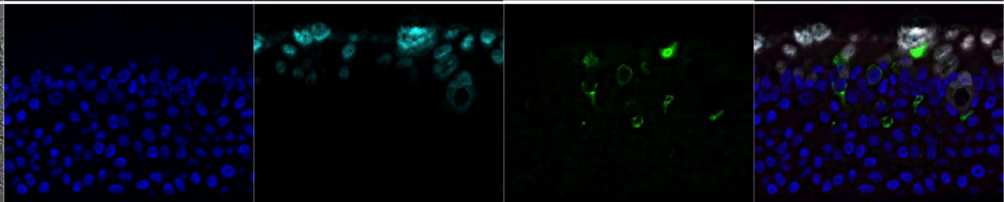

Increased
squamous metaplasia

D

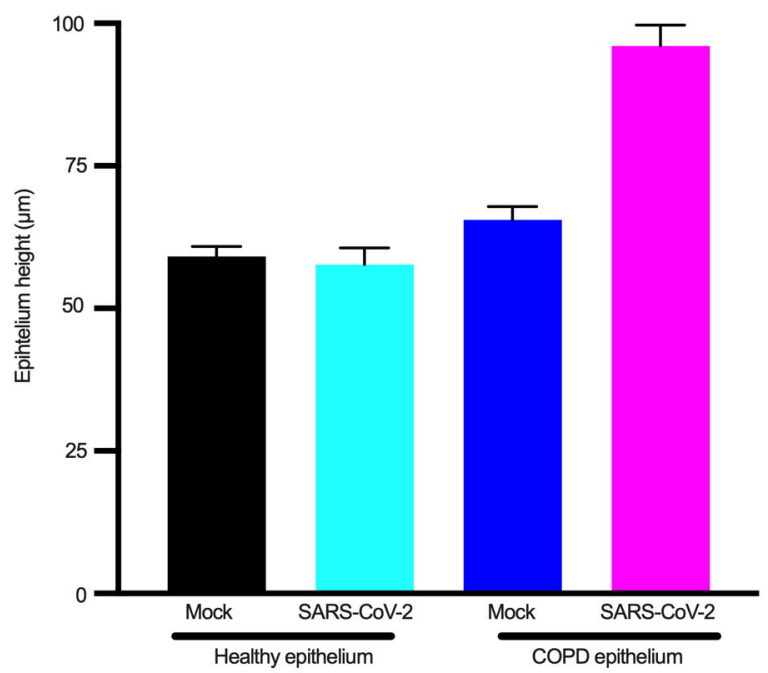

Figure 6 
A

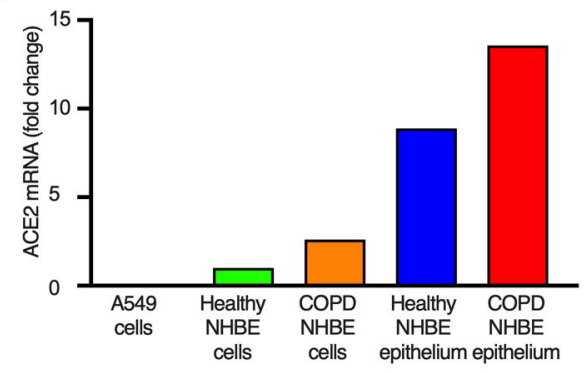

B

Healthy COPD

epithellium epithelium

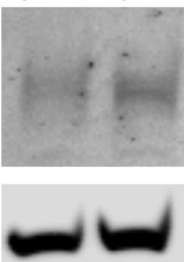

C

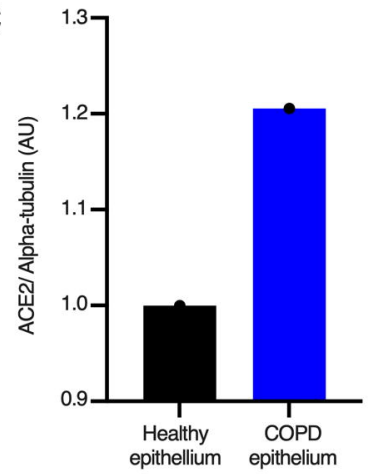

D

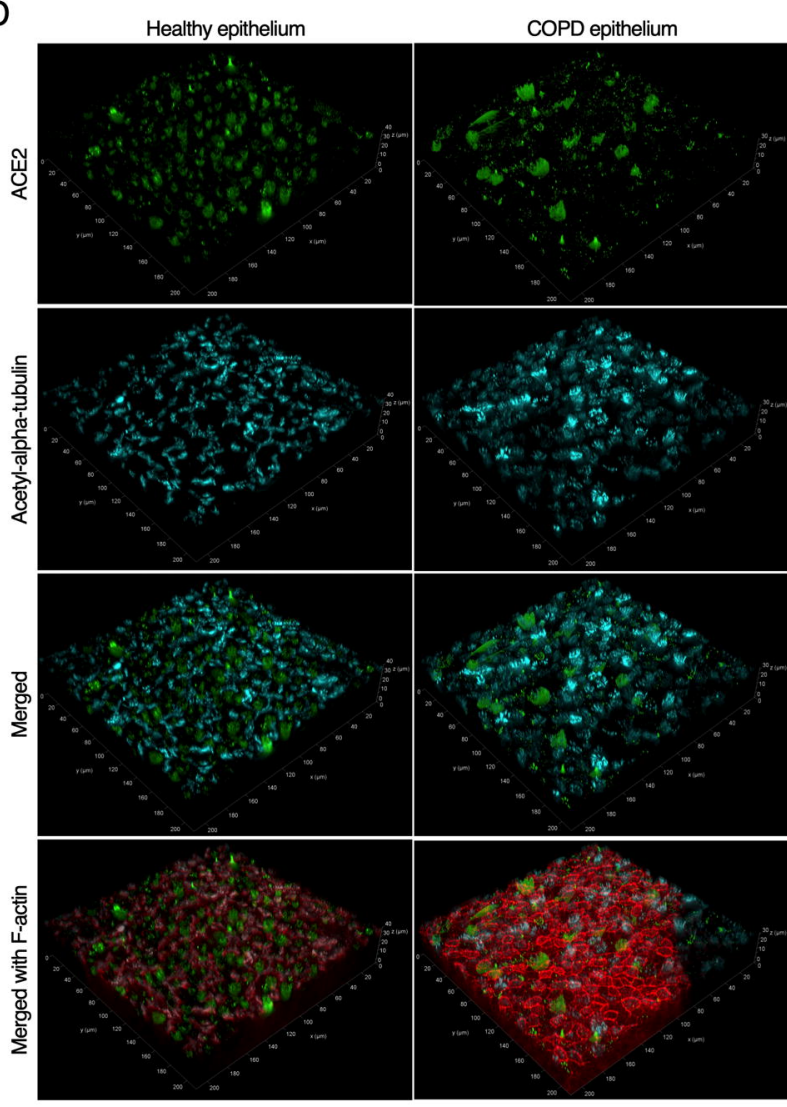

Healthy epithelium

F

Alpha-tubulin
ACE2

$$
\text { ్ㅣㄴ }
$$

Healthy epithelium

COPD epithelium

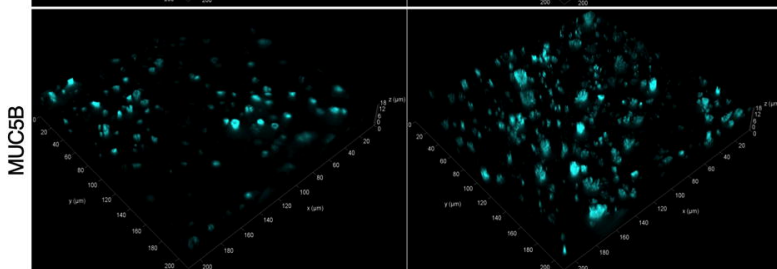

할

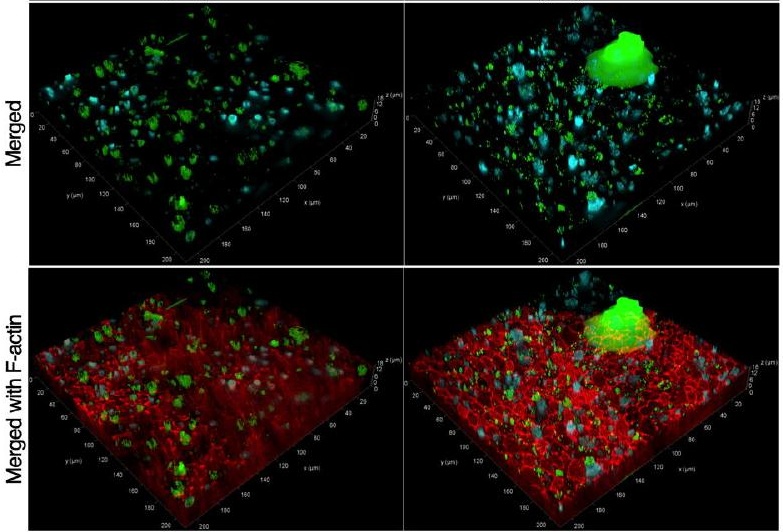

E

$\mathrm{E}$

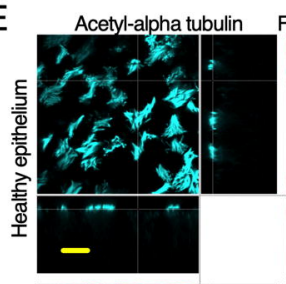

$\mathrm{F}$-actin + Acetyl-alpha tubulin

ACE2
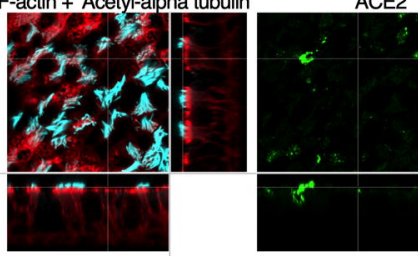

F-actin + ACE2

Merged with nucleus
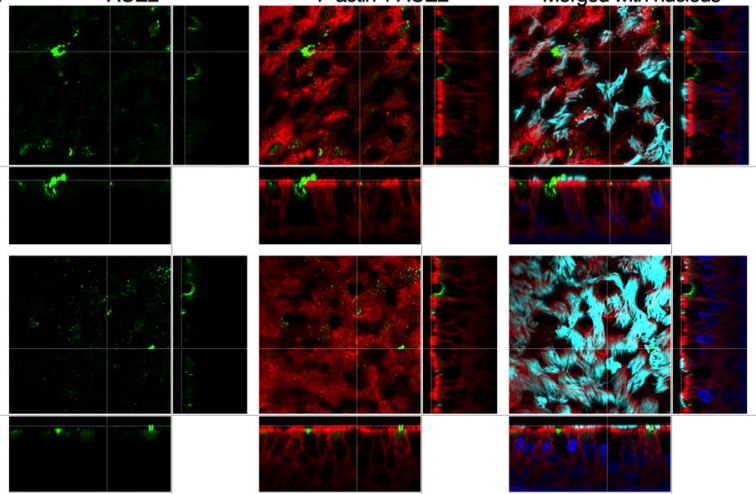

Figure S1 


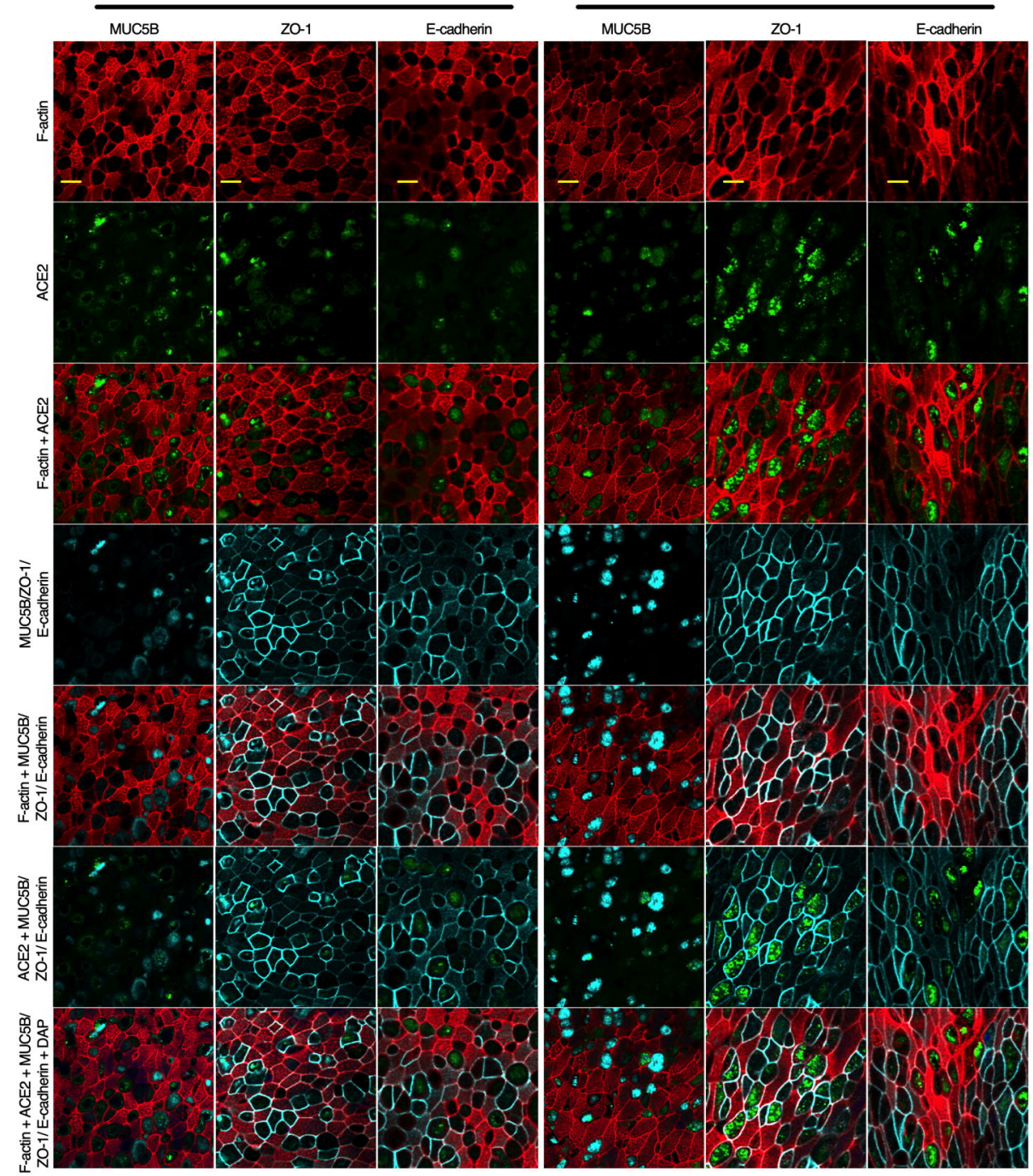


A Acetyl-alpha tubulin

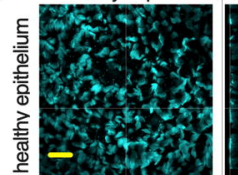

항
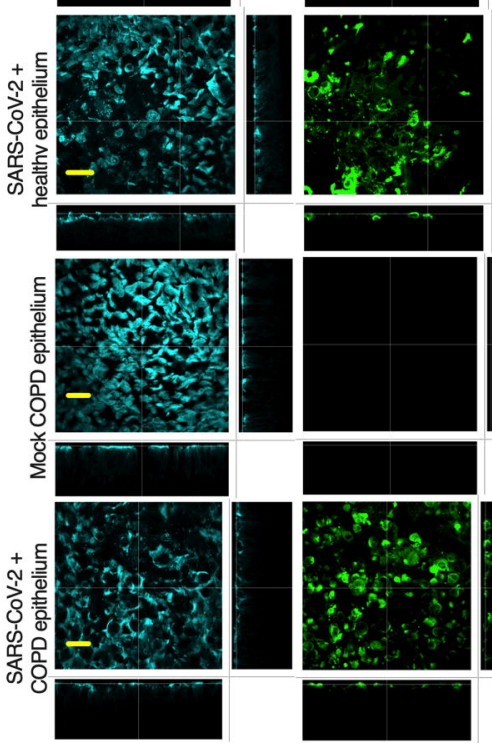

B

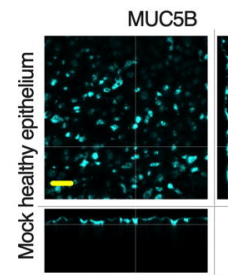

$+$

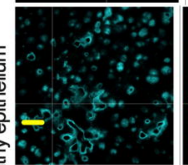

क
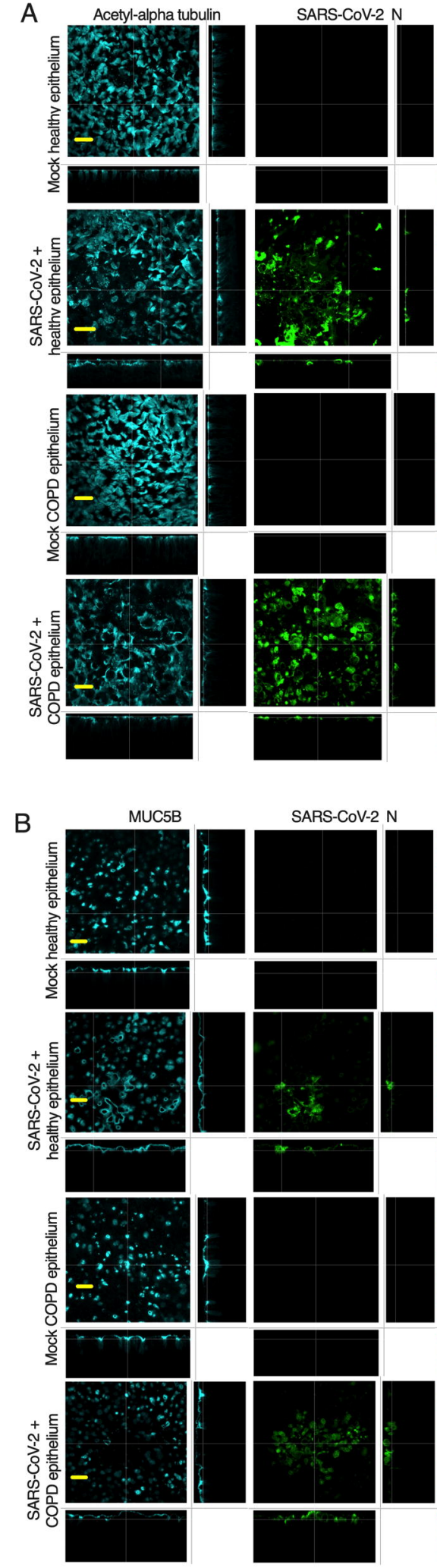
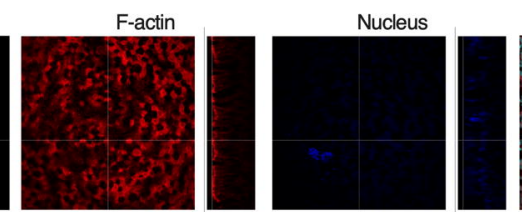

Merged
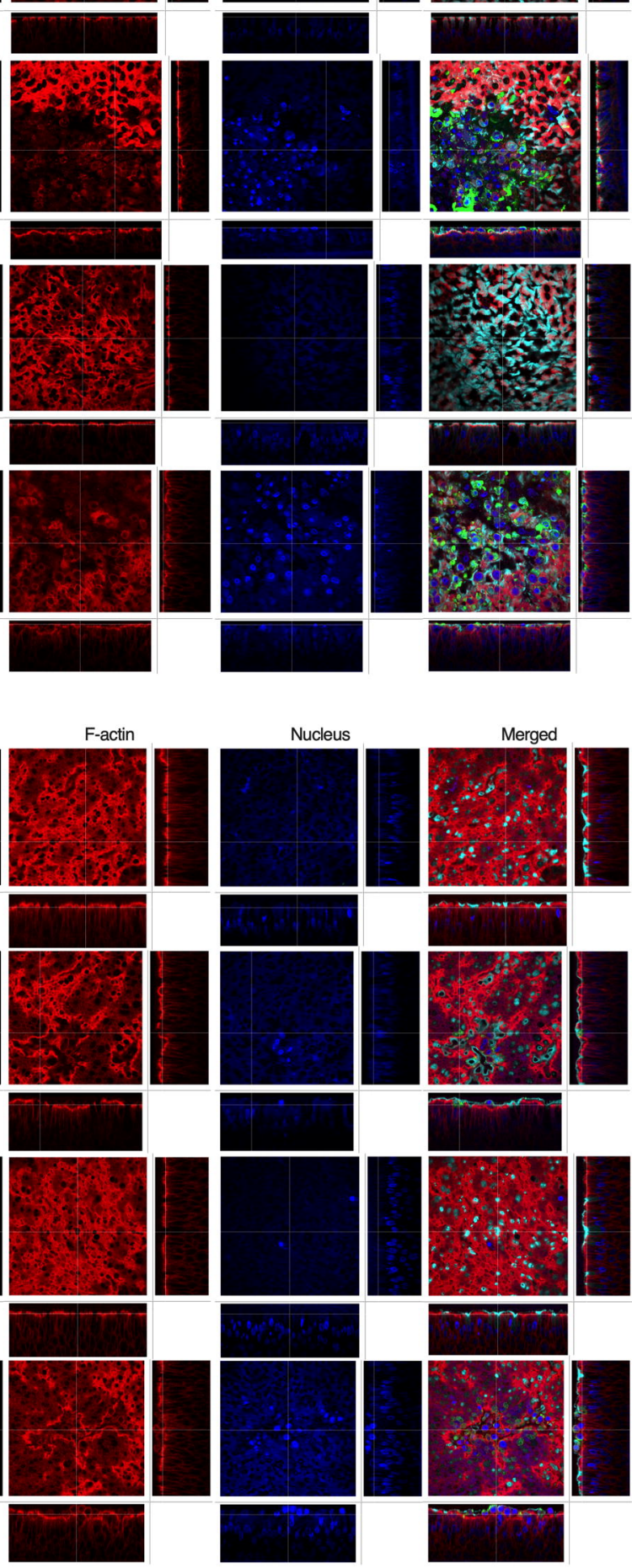

Figure S3 
Mock-infected

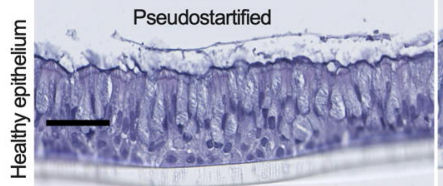

SARS-CoV-2 infected, 4 DPI
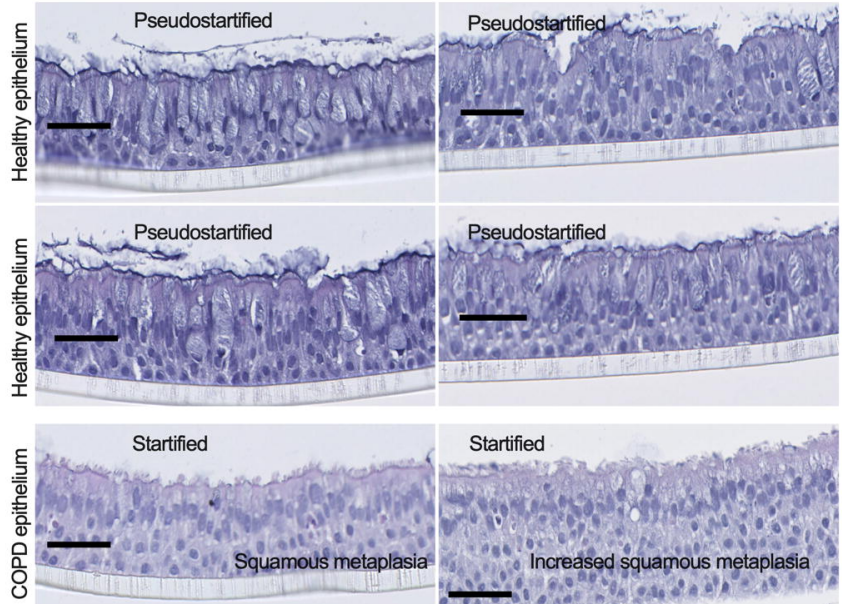

\section{Startified}

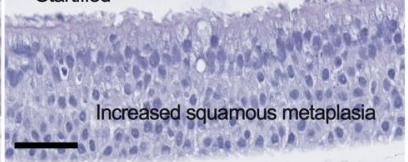

\section{Startified}

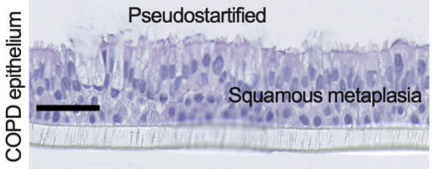

\section{Figure S5}


Healthy Epithelium
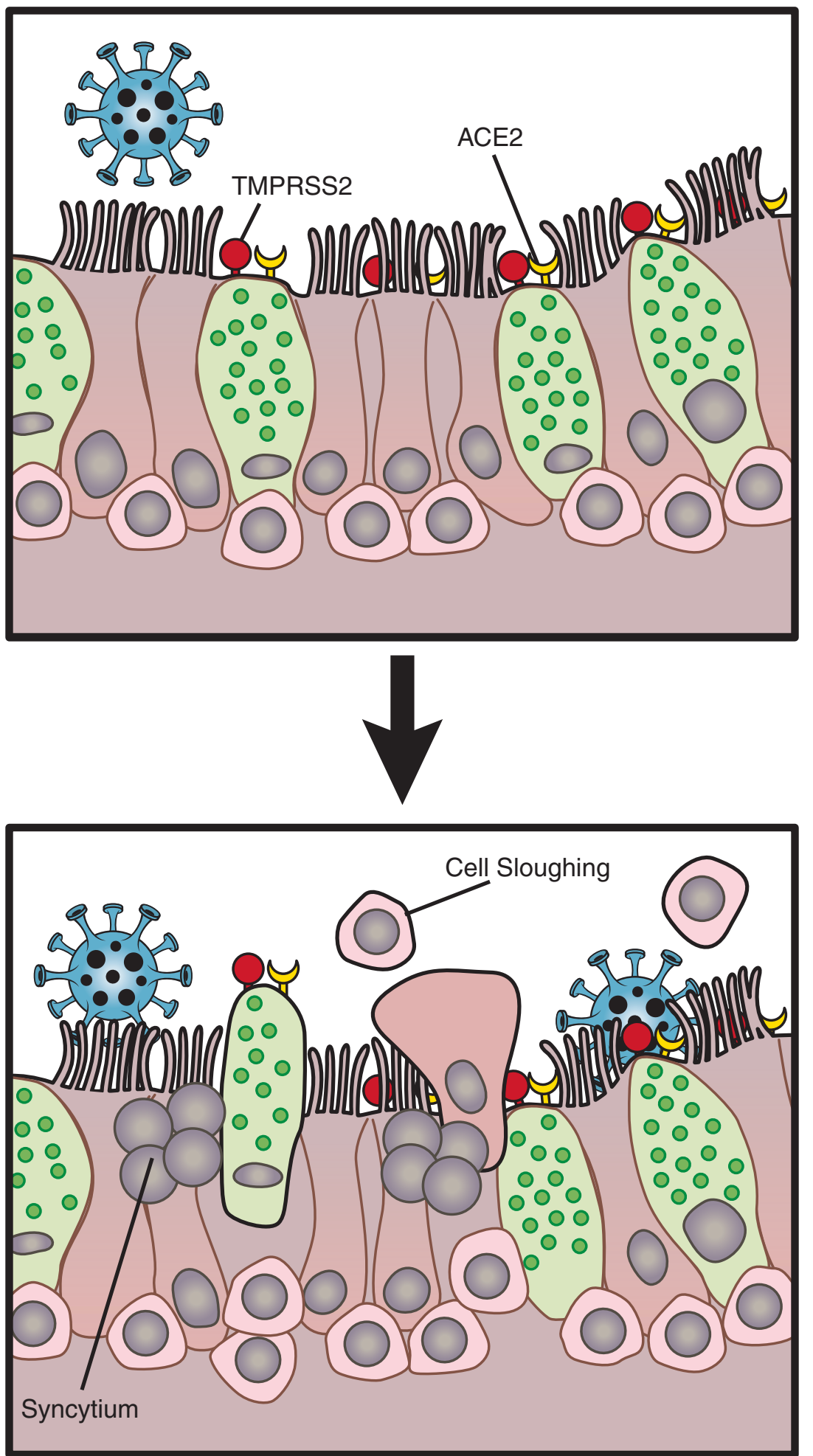

COPD Epithelium
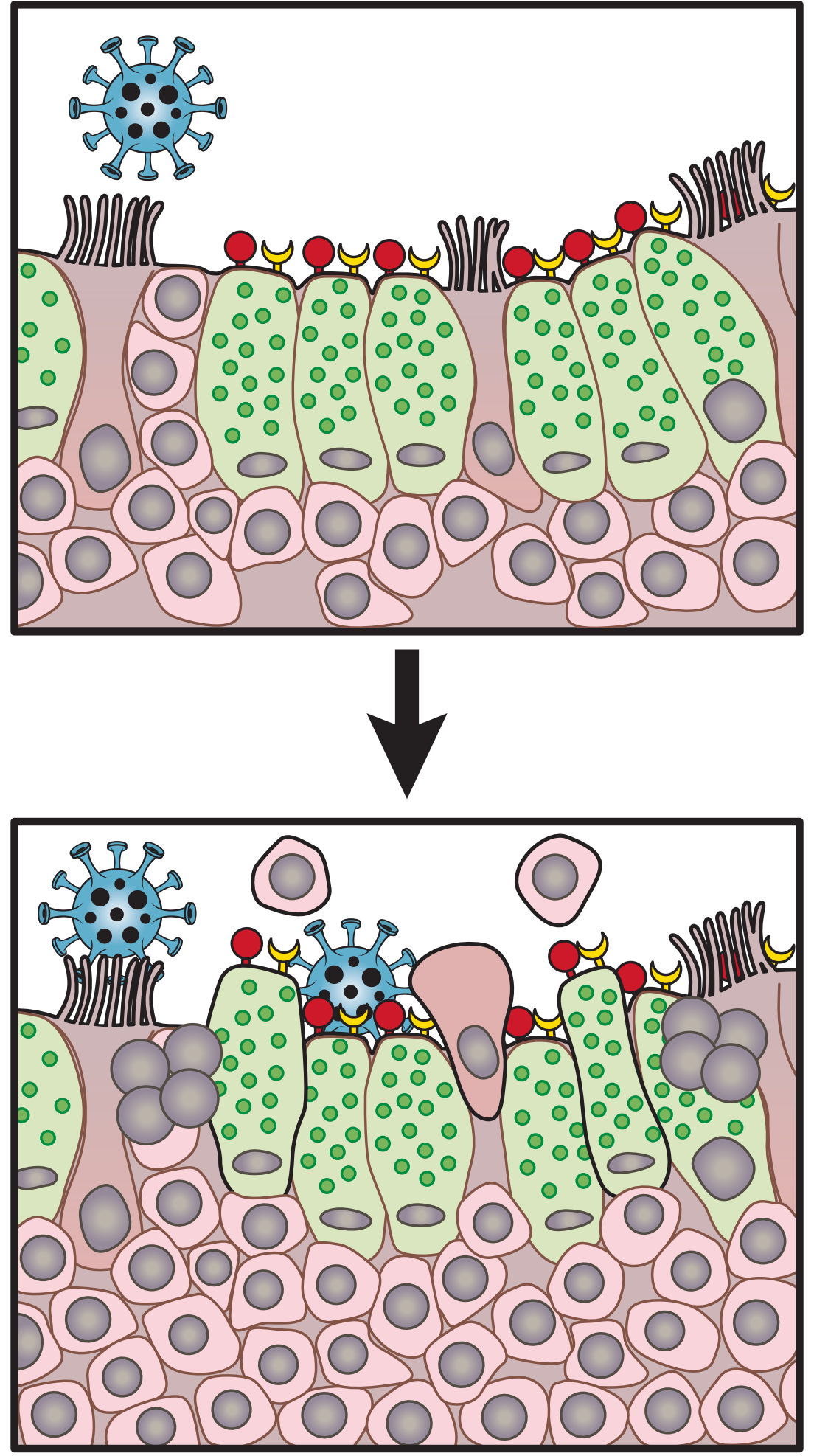